\title{
Proximal CA3 is the primary locus of age-related pattern separation deficits in rats
}

Heekyung Lee ${ }^{1}$, Arjuna Tilekeratne ${ }^{1}$, Nick Lukish ${ }^{1}$, Zitong Wang $^{2}$, Scott Zeger ${ }^{2}$, Michela

Gallagher $^{3}$, James J. Knierim ${ }^{1,3,4}$

${ }^{1}$ Krieger Mind/Brain Institute, Johns Hopkins University, Baltimore, MD, 21218

${ }^{2}$ Department of Biostatistics, Johns Hopkins Bloomberg School of Public Health, Baltimore, MD, 21205

${ }^{3}$ Department of Psychological and Brain Sciences, Johns Hopkins University, Baltimore, MD

${ }^{4}$ Solomon H. Snyder Department of Neuroscience, Johns Hopkins School of Medicine,

Baltimore, MD, 21205

Correspondence: heekyung@jhu.edu; jknierim@jhu.edu

pg. 1 


\begin{abstract}
Age-related deficits in pattern separation have been postulated to bias the output of hippocampal memory processing toward pattern completion, which can cause deficits in accurate memory retrieval. While the CA3 region of the hippocampus is often conceptualized as a homogeneous network involved in pattern completion, growing evidence demonstrates a functional gradient in CA3 along the transverse axis, with proximal CA3 supporting pattern separation and distal CA3 supporting pattern completion. We examined the neural representations along the CA3 transverse axis in young $(Y)$, aged memory-unimpaired $(A U)$, and aged memory-impaired $(\mathrm{Al})$ rats when different changes were made to the environment. When the environmental similarity was high (e.g., altered cues or altered environment shapes in the same room), $\mathrm{Y}$ and $\mathrm{AU}$ rats showed more orthogonalized representations in proximal CA3 than in distal CA3, consistent with prior studies showing a functional dissociation along the transverse axis of CA3. In contrast, Al rats showed less orthogonalization in proximal CA3 than $\mathrm{Y}$ and $\mathrm{AU}$ rats but showed more normal (i.e., generalized) representations in distal CA3, with little evidence of a functional gradient. When the environmental similarity was low (e.g., recordings were done in different rooms), representations in proximal and distal CA3 remapped in all rats, showing that $\mathrm{Al}$ rats are able to dissociate representations when inputs show greater dissimilarity. These results provide evidence that the aged-related bias towards pattern completion is due to the loss in Al rats of the normal transition from pattern separation to pattern completion along the CA3 transverse axis and, furthermore, that proximal CA3 is the primary locus of this age-related dysfunction in neural coding.
\end{abstract}

Keywords: CA3; aging; hyperactivity; pattern separation; pattern completion 


\section{Introduction}

Aging increases the susceptibility to memory interference (Ebert \& Anderson, 2009; Emery et al., 2008; Lustig \& Hasher, 2001), which occurs when similar memories compete with each other and impair the correct retrieval of any one of those memories. To minimize memory interference and maximize storage capacity in a distributed memory system, computational models hypothesized that the hippocampus performs two complementary processes: pattern separation (the ability to orthogonalize similar input patterns to create less overlapping output patterns) and pattern completion (the ability to retrieve stored output patterns when presented with partial or degraded input patterns) (Marr, 1971; McClelland \& Goddard, 1996; McNaughton \& Morris, 1987; O’Reilly \& McClelland, 1994; Rolls \& Treves, 1998; Treves \& Rolls, 1992).

Place cell remapping is often used as a proxy to study pattern separation. Place cells are hippocampal neurons that fire when an animal occupies a specific location in an environment (O'Keefe \& Dostrovsky, 1971) and are proposed to create a cognitive map that stores experiences associated with that environment (O'Keefe \& Nadel, 1978). Place cells undergo remapping when changes are made to the environment or when the animal is moved to a different environment (Muller \& Kubie, 1987), as well as when the animal changes its behavior or internal state (Ferbinteanu \& Shapiro, 2003; Markus et al., 1995; Moita et al., 2004; Wood et al., 2000). In remapping, place fields can appear, disappear, change firing locations, or change firing rates (Anderson \& Jeffery, 2003; Leutgeb et al., 2005; Muller \& Kubie, 1987). Thus, remapping creates dissimilar spatial maps for distinct experiences or contexts that can be stored as different memories.

Converging studies from rodents and humans have demonstrated an age-related decline in pattern separation abilities. Rodent studies have shown that CA3 place cells of aged rats failed to encode changes in the environment, demonstrating a rigidity in their representations (Robitsek et al., 2015; Wilson et al., 2005). Similarly, in human fMRI studies, representational rigidity in older adults' DG/CA3 region was linked to mnemonic discrimination deficits (Lacy et 
al., 2011; Yassa et al., 2010, 2011). Studies of aged CA3 have indicated that hyperactivity contributes to pattern separation deficits by strengthening the autoassociative CA3 network to favor enhanced pattern completion (Haberman et al., 2017; H. Lee et al., 2021; Maurer et al., 2017; Robitsek et al., 2015; Thomé et al., 2016; Wilson et al., 2005; Yassa et al., 2011). Accordingly, treatments with the antiepileptic drug, levetiracetam, to reduce hyperactivity, resulted in improved memory performance in aged rats (Haberman et al., 2017; Koh et al., 2010; Robitsek et al., 2015) and in amnestic mild cognitive impairment patients (Bakker et al., 2012, 2015), suggesting that normalizing CA3 hyperactivity restored the balance between pattern separation and pattern completion.

Anatomical and functional heterogeneity along the CA3 transverse axis suggests that proximal CA3 supports pattern separation and distal CA3 supports pattern completion (Hunsaker et al., 2008; Ishizuka et al., 1990; H. Lee et al., 2015; Lu et al., 2015; Marrone et al., 2014; Nakamura et al., 2013; Sun et al., 2017; Witter, 2007). Because age-related hyperactivity appears restricted to proximal CA3, while potential hypoactivity is present in distal CA3 $(\mathrm{H}$. Lee et al., 2021), the age-related bias from pattern separation to pattern completion may be specific to the proximal CA3 region. However, there have been no direct studies examining the neural representations along the functionally graded CA3 axis in aging. The present study aims to determine how age-related dysfunctions result from an imbalance of the normal gradient of pattern separation and pattern completion along the CA3 transverse axis.

\section{Results}

Histology and experimental procedure

Multielectrode recordings were made along the dorsal CA3 transverse axis (Fig. 1A, Supp. Fig.1) in 8 young and 14 aged rats. All aged rats and 4 of 8 young rats were behaviorally characterized in the Morris water maze (see Methods), and the aged rats were categorized as 
learning impaired $(\mathrm{Al} ; \mathrm{n}=7)$ or learning unimpaired $(\mathrm{AU} ; \mathrm{n}=7)$ based on pre-determined learning index (LI) scores (Branch et al., 2019; Gallagher et al., 1993) (Fig. 1B). Four of 8 young rats were not pretested in the Morris water maze and were part of a previous study $(\mathrm{H}$.

Lee et al., 2015). Three experiments were performed. In the cue-conflict experiment, all 22 rats were trained to run clockwise on a circular track located in a curtained room (Fig. 1C). Four different local textures tiled the surface of the circular track and multiple global cues were placed along the curtained room (Knierim, 2002). Three standard (STD) sessions with a fixed local and global cue configuration were interleaved with two cue-mismatch (MIS) sessions, in which the track was rotated in a counterclockwise (CCW) direction and the global cues in a clockwise (CW) direction. In the two-shape experiment, subsets of the rats $(3 \mathrm{Y}, 6 \mathrm{AU}$, and $6 \mathrm{Al}$, all of which were behaviorally characterized in the Morris water maze) were trained to forage in circle and square enclosures placed at a constant location in room A. In the two-room experiment, the same subsets of rats were taken to room B (after the four sessions of alternating square and circle in room A) and two more sessions were recorded in the circle and square enclosures placed at a constant location in room B. 
A)

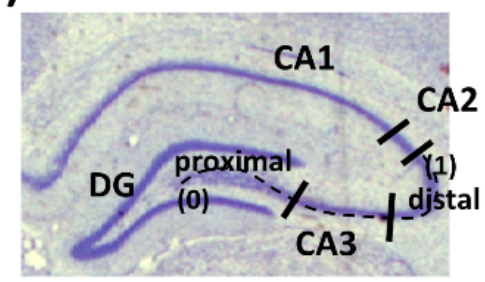

B)

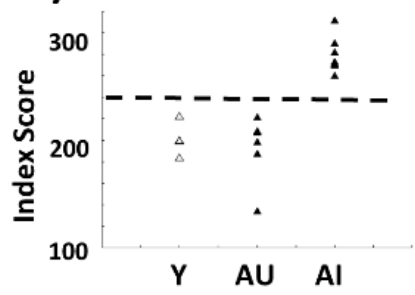

Y AU Al

C)

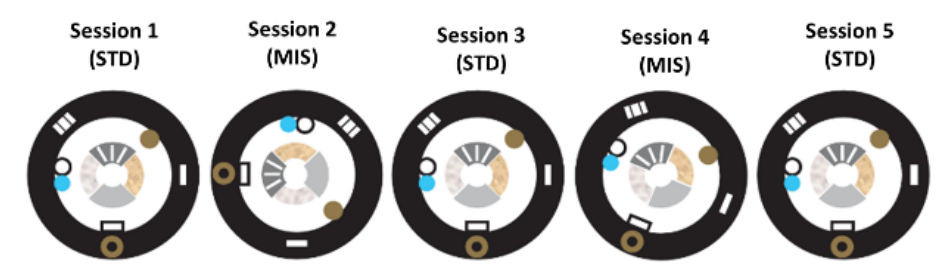

D)

Room A
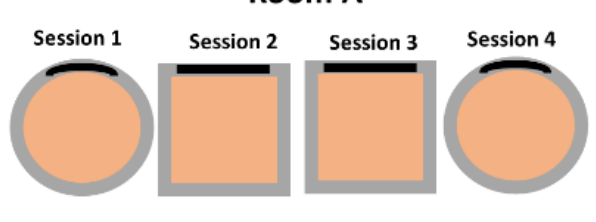

Room B

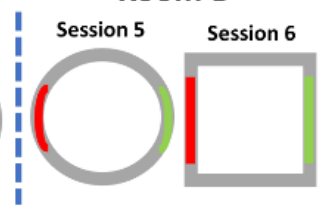

Figure 1. CA3 recording locations and experimental procedures. (A) Recordings were made along the CA3 transverse axis. The length of the CA3 transverse axis was normalized from 0 to 1 , with the proximal region defined as locations between 0 and 0.4 , the intermediate region from 0.4 to 0.7 , and the distal region from 0.7 to 1 of the axis length (Lee et al., 2021). (B) A learning index score was derived from measures of proximity to the goal location during probe trials interpolated throughout the water maze training, with lower scores indicating more accurate performance. Aged rats that performed on par with young rats were designated as aged-unimpaired $(\mathrm{AU})$, and those that performed more poorly than young rats were designated as aged-impaired (Al), according to established criteria (Gallagher et al., 1993). (C) In the local-global cue mismatch experiment, recording sessions consisted of three standard (STD) sessions interleaved with two mismatch (MIS) sessions. In the STD sessions, the local cues on the track (denoted by the inner ring with four different textures) and the global cues along the curtain at the periphery (denoted by the black outer ring) were arranged in a fixed configuration that the rat had experienced during training periods. In the MIS sessions, the global cues were rotated in a clockwise direction and the local cues were rotated in a counterclockwise direction by the same amount, for a net mismatch of $90^{\circ}, 135^{\circ}$, or $180^{\circ}$. (D) In the two-shape experiment, recording sessions consisted of 4 sessions (two sessions in a circle and two sessions in a square) in room $\mathrm{A}$. In the two-room experiment, after 4 sessions in room A, two additional sessions (one session each in circle and square) were recorded in room B. In room A, there was a striped cue card on the north wall of the circle and square enclosures placed above a brown paper floor. In room B, there were two cue cards (a brown cue card on the west and a gray cue card on the east) on the walls of the enclosures placed above a white paper floor. 
Individual cell responses in the cue-conflict manipulations along the CA3 transverse axis

In the cue-conflict experiment, individual cell responses to the cue manipulations were categorized into five groups, similar to previously published studies using the same task $(\mathrm{H}$. Lee et al., 2015; I. Lee et al., 2004; Neunuebel \& Knierim, 2014) (Fig. 2A). Classifications were based on each cell's response in the MIS session compared to the cell's firing properties in the immediately preceding STD session. For simplicity, the 5 categories were condensed into 2 categories, such that if a cell met the place-field inclusion criteria (spatial information score > 0.75 , significance $p<0.01$, number of on-track spikes $>50$ ) in both STD and MIS sessions, the cell was classified as a "Rotate" cell. If a cell met the place-field inclusion criteria in either the STD (i.e., place field DISAPPEARED) or MIS (i.e., place field APPEARED) session, but not in both, the cell was classified as a "Remap" cell. Because previous studies in this cue-mismatch experiment have shown that the large majority of Rotate cells are controlled strongly by the local or global sets of cues (Knierim, 2002; H. Lee et al., 2015; I. Lee et al., 2004; Neunuebel \& Knierim, 2014), we consider this class as approximately indicating the degree to which the hippocampus maintains the same representation between the two sessions. In contrast, cells that are classified as Remap indicate the degree to which the hippocampus creates different representations between the environments.

There were notable group differences across the CA3 subregions in the proportion of the response types (Fig. 2B, Supp. Table 1). In Y rats, there was a decreasing proportion of Remap cells, with a correspondingly increasing proportion of Rotate cells, from proximal to distal CA3. This trend is consistent with previous findings in $Y$ rats reported by Lee et al. (2015). AU rats showed a similar trend as $Y$ rats, although the proportions in each region were different from $Y$ rats. However, the trend was opposite in Al rats, with a decreasing proportion of Rotate cells and an increasing proportion of Remap cells from proximal to distal CA3. Specifically, in the $Y$ rats, a high proportion (75\%) of cells in proximal CA3 remapped $(n=156 / 207)$, compared to $65 \%$ of cells in intermediate CA3 $(n=126 / 195)$ and $56 \%$ of cells in distal CA3 $(n=67 / 120)$. In 
AU rats, $57 \%$ of cells in proximal CA3 remapped $(n=81 / 142)$, compared to $48 \%$ in intermediate CA3 $(n=52 / 109)$, and $39 \%$ in distal CA3 $(n=18 / 46)$. In contrast, in Al rats, only $28 \%$ of cells remapped in proximal CA3 $(n=24 / 85), 35 \%$ in intermediate CA3 $(n=58 / 165)$, and $43 \%$ in distal CA3 $(n=20 / 47)$. There was a significant association between the proportion of response types and the age groups in the proximal $\left(X^{2}{ }_{(2,434)}=56.67, \mathrm{p}<0.0001\right)$ and intermediate $\left(X^{2}(2,469)=\right.$ 31.42, $\mathrm{p}<0.0001)$ regions but not in the distal region $\left(X^{2}(2,213)=4.81, p=0.09\right)$, suggesting response types differ among the age groups in proximal and intermediate CA3 but not in distal CA3 (see below for statistical evaluations of an interaction between age and CA3 region).

A)

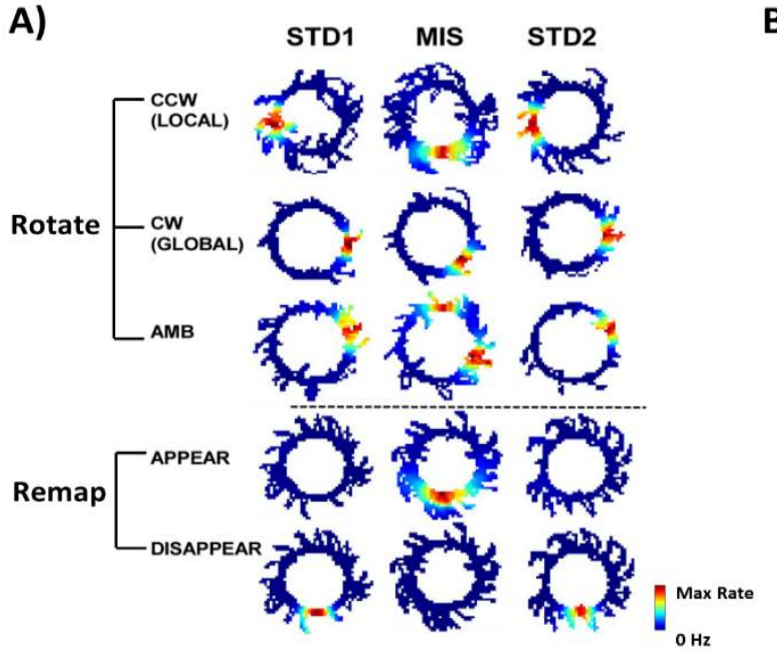

B)

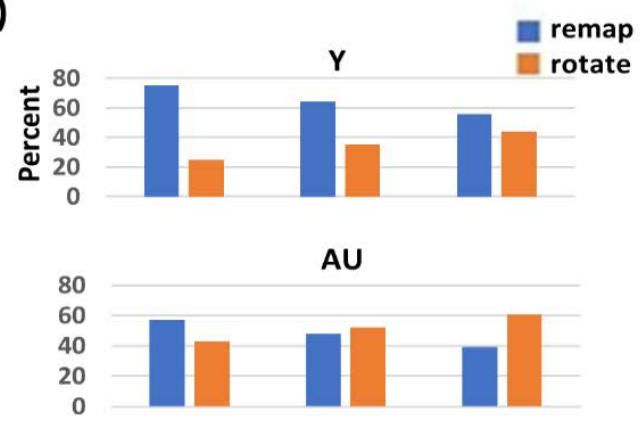

Al

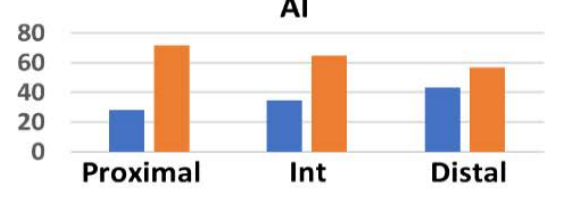

Figure 2. Responses to the cue manipulations differ in age groups along the CA3 transverse axis. (A) Examples of five different response types observed in CA3. Cells that showed CCW rotation with the local cues, CW rotation with the global cues, and ambiguous (AMB) rotation were categorized as "Rotate" cells. For example, compared to the location of the place field in STD1, the location of the place field in MIS rotated in either a CCW or a CW direction. In AMB rotation, a single place field in STD1 split into two fields in MIS, with one field showing CCW rotation and the other field showing CW rotation. Cells with place fields that APPEAR in MIS or DISAPPEAR in MIS were categorized as "Remap" cells. (B) Classification of responses in different CA3 subregions are different in age groups. $\mathrm{Y}$ and $\mathrm{AU}$ rats showed a similar trend of a decreasing proportion of Remap cells from proximal to distal CA3, with an increasing proportion of Rotate cells from proximal to distal CA3. In contrast, Al rats showed an opposite trend, with a decreasing proportion of Rotate cells from proximal to distal CA3 and an increasing proportion of Remap cells from proximal to distal CA3. 
Population responses in the cue-conflict manipulations along the CA3 transverse axis

To quantitatively analyze population responses, spatial correlation matrices from the population firing rate vectors at each location on the circular track were created $(\mathrm{H}$. Lee et al., 2015; I. Lee et al., 2004; Neunuebel \& Knierim, 2014) (Fig. 3A). The spatial correlation matrices reflect whether the population of cells rotated their firing locations coherently. To create these matrices, the firing rate of each cell was calculated for each $1^{\circ}$ bin on the track and normalized to its peak rate. The firing rate maps of all $n$ cells in the sample were stacked to create a $360 \mathrm{x}$ $n$ matrix, in which each column of the matrix represents the population firing rate vector for each angle of the track (Supp. Fig. S2). The firing rate vectors at each angle of a STD session (STD1) were correlated with the firing rate vectors at each angle of the next STD session (STD2), to create STD1 x STD2 correlation matrices, or with the next MIS session, to create STD1 x MIS correlation matrices. To make statistical comparisons across age groups and CA3 subregions tractable, we collapsed the data from all 3 rotation mismatch amounts. Because prior studies of CA3 with this same cue-mismatch manipulation (H. Lee et al., 2015; I. Lee et al., 2004; Neunuebel \& Knierim, 2014) have shown that place fields tended to be controlled by the local cues (i.e., the place fields rotated along the track by increasing amounts when the local cues were rotated by increasing amounts; Supp Fig. S3), we first adjusted the firing rate maps to compensate for the different degrees of cue rotation. To do so, the mean rotation angle of place fields for all recorded cells of a MIS amount $\left(90^{\circ}, 135^{\circ}\right.$, or $\left.180^{\circ}\right)$ was calculated (Supp. Fig. S2). Each cell's firing rate map in each MIS session was then rotated by this mean rotation amount. Thus, if the place fields rotated coherently in the MIS sessions, controlled by one set of cues, rotating the rate maps in the MIS session by the mean rotation amount would align the location of the place fields in MIS sessions with the location of the place fields in the preceding STD1 session. This alignment would create a strong correlation band along the main diagonal in the spatial correlation matrices between the STD and MIS sessions. However, if the cells did not rotate coherently in the MIS sessions or if the cells remapped, rotating the place fields in the 
MIS session by the mean rotation amount would misalign the locations of the place fields in MIS and STD sessions, creating a dispersed and weak correlation band along the main diagonal in the spatial correlation matrices between the STD and MIS sessions.

Figure 3A demonstrates the population coherence between repeated sessions in the STD sessions and between the STD and MIS sessions. Columns 1, 3, and 5 show correlation matrices for STD1 vs. STD2 sessions for all age groups and CA3 regions. In each case, there is a band of high correlation along the main diagonal of the matrix (dashed line), indicating that the population representation of spatial location on the track was similar (i.e., coherent) between the two sessions. Columns 2, 4, and 6 show correlation matrices between the STD and MIS sessions. For $\mathrm{Y}$ rats, the correlation band along the main diagonal of proximal CA3 was very weak, indicating that there was a large amount of spatial remapping between the two sessions. For intermediate and distal CA3, however, the correlation band was stronger, replicating the result of Lee et al. (2015) of a gradient from pattern separation to pattern completion along the transverse axis of CA3. Both $\mathrm{AU}$ and $\mathrm{Al}$ rats showed stronger proximal CA3 correlation bands than $Y$ rats, and less of a difference in the strength of the bands from proximal to distal CA3.

To quantitatively analyze the strengths of the correlation bands between age groups and between CA3 subregions, the correlations along the main diagonals of the matrices were compared. It is possible that the different age groups and CA3 subregions may have different degrees of stability of their spatial maps even between 2 experiences in the same environment. To control for such inherent variability, the correlation differences between the main diagonals of the STD matrix (STD1 $x$ STD2) and the MIS matrix (STD1 x MIS matrix) were calculated for each animal group in each subregion (Supp. Fig. S2). Thus, if the correlation strength in the STD matrix is stronger than the correlation strength in the MIS matrix, the correlation difference will be high. If the correlation strengths between the two matrices are similar, the correlation difference will be low. There was a decreasing trend in the correlation difference from proximal to distal in $\mathrm{Y}$ and $\mathrm{AU}$ rats, suggesting that the correlations in the MIS matrices became stronger 
from proximal to distal CA3 (Fig. 3B). In contrast, the trend was opposite in Al rats, suggesting that the correlations in the MIS matrices were stronger in proximal but became weaker in distal CA3.

To statistically compare the magnitude of correlation differences between the age groups in each subregion, the correlation difference of one age group in one subregion was subtracted from that of the other age group (Fig. 3C). For age groups being compared in each subregion, the statistical significance was calculated by a shuffling procedure in which the cells from both age groups (e.g., Y vs. Al) were randomly reassigned to each age group. The correlation difference was then calculated from the shuffled dataset. This procedure was repeated 1000 times to create a simulated distribution based on the random shuffling of the data. In proximal CA3, there were significant differences in correlation strengths between $\mathrm{Y}$ and Al rats and between $\mathrm{Y}$ and $\mathrm{AU}$ rats (i.e., real data point [thick black line] was outside the 95\% confidence interval [dotted black lines] of the simulated distributions with significance level $\alpha=$ 0.005, with Bonferroni correction for 9 comparisons) but not in intermediate and distal CA3. There were no significant differences between $\mathrm{AU}$ and $\mathrm{Al}$ rats in any CA3 subregions.

The above results suggest that there is an interaction between age group and CA3 subregion, in that aged rats show less pattern separation than $Y$ rats in proximal CA3 but not in distal CA3. To statistically analyze this interaction, for each between-age group comparisons of Fig 3C, we calculated the correlation difference between each subregion (Fig. 3D). A significant interaction was seen between $\mathrm{Y}$ and both sets of aged rats in proximal and distal subregion comparisons. From these tests (Figs 2-3), we can conclude that AU and Al rats show more highly correlated representations between STD and MIS sessions (i.e., less pattern separation) in proximal CA3 than $\mathrm{Y}$, but similarly correlated representations in distal CA3 (see also Supp Fig. S4, S5). 
A)

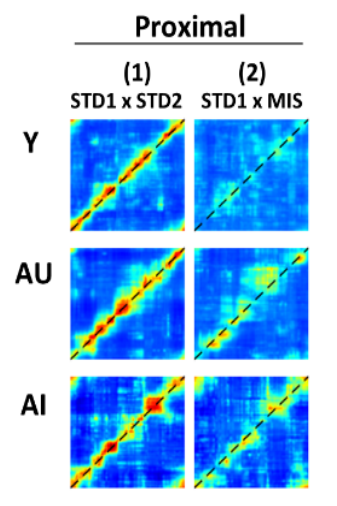

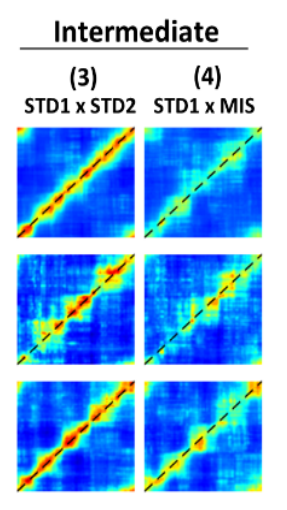

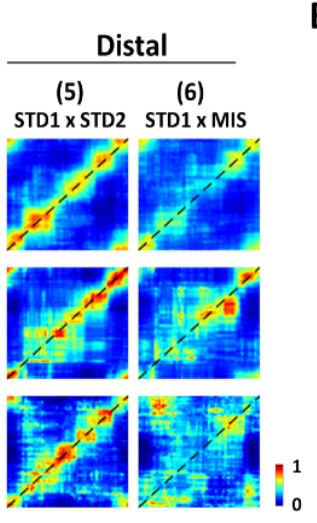

B)

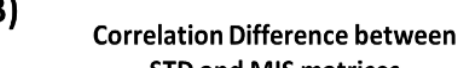
STD and MIS matrices

C)
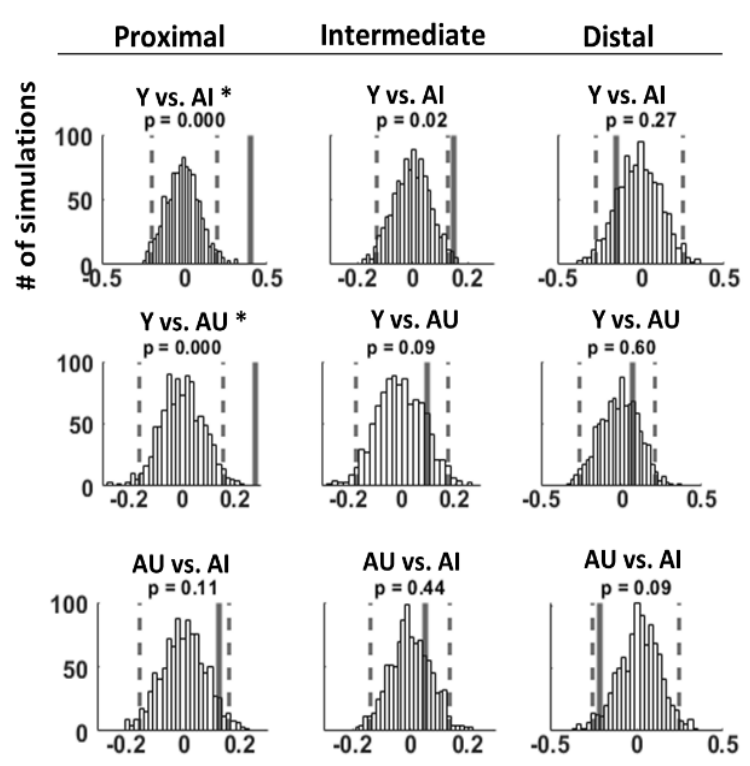

Normalized Sum Correlation Difference
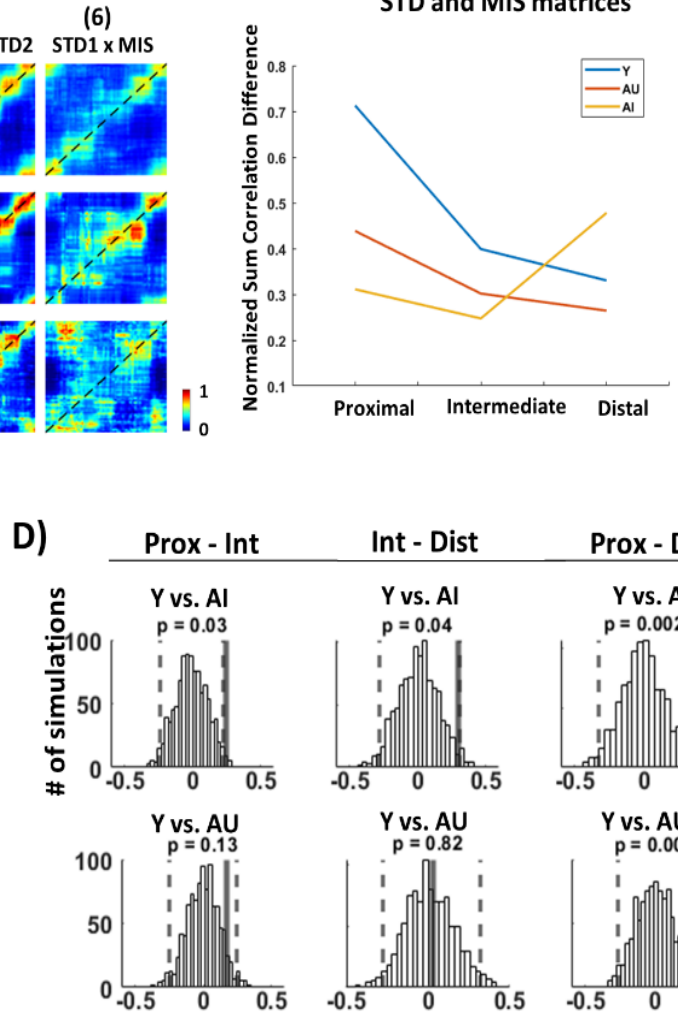

Figure 3. Shuffling analyses show that correlated representations between the STD and MIS sessions differ in age groups along the transverse axis. (A) STD1 $\times$ STD2 matrices (columns 1, 3, 5) and STD1 x MIS matrices (columns 2, 4, 6) in each CA3 subregions for all age groups. (B) The correlation difference between STD and MIS matrices showed a decreasing trend in $Y$ and $A U$ rats from proximal to distal CA3 but an increasing trend in Al rats, indicating an increase in population coherence (pattern completion) from proximal to distal $C A 3$ in $Y$ and $A U$ rats and a corresponding decrease in Al rats. (C) Comparisons between the age groups were calculated for each subregion (top row: $Y-A l$; middle: $Y-A U$; bottom: $A U-A l$ ). The observed value (thick, black line) was compared to the distribution produced by 1,000 random shuffles of the data. Dashed lines indicate the $2.5^{\text {th }}$ and $97.5^{\text {th }}$ percentiles of the distribution. (D) To test for interactions between age and subregions, comparisons between the subregions were calculated for each between-age group comparisons. * denotes significance at $p=0.005$, Bonferroni corrected for 9 comparisons. 


\section{Global-cue rotation only in CA3}

Since the MIS sessions require recognition of a mismatch between the global and the local cues, a control experiment was performed to verify that aged rats were able to see the global cues. The textured track was replaced with a solid wooden track to remove the salient, local texture cues on the track. All rats were then given STD1-MIS-STD2 sessions where only the global cues were rotated by $45^{\circ}$ or $90^{\circ}$ in the clockwise direction in the MIS session. Population coherence demonstrated global cue control when local cues were removed (Supp. Fig. S6), suggesting that the aged rats could perceive the global cues and that the place cells can be controlled by the global cues in the absence of conflicting local-cue information (Knierim \& Hamilton, 2011).

\section{Two-shape experiment}

To test whether the interaction between the age groups and CA3 subregions seen in the cue-mismatch experiment generalized to other conditions of remapping, a subset of rats from the cue-mismatch experiment were recorded during open-field foraging in two different shapes, a square (S) and a circle (C) enclosure, placed at a constant location in room $A$ (none of the rats came from prior studies). Three $\mathrm{Y}$ (112 cells meeting place-field criteria), $6 \mathrm{AU}$ (142 cells), $6 \mathrm{Al}$ (131 cells) (Supp. Table 2) were recorded for two days with the session sequence as either SC-C-S or C-S-S-C. Example rate maps along the CA3 transverse axis showed a marked difference among the age groups (Fig. 4). Rate maps for $Y$ rats showed that place cells remapped between the two shapes (i.e., fired only in one shape) in proximal and intermediate CA3 but fired in both shapes in distal CA3. Rate maps for AU rats showed that place cells remapped in proximal but fired in both shapes in intermediate and distal CA3. In contrast, place cells in Al rats fired in both shapes in all CA3 subregions. 
A)

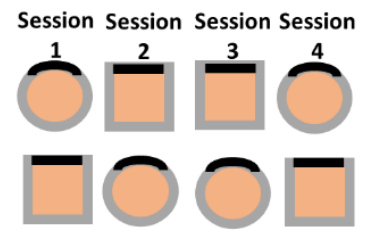

B)

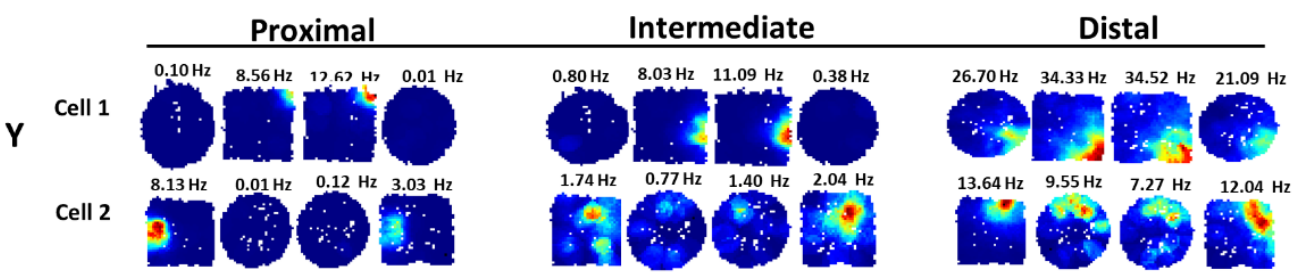

AU
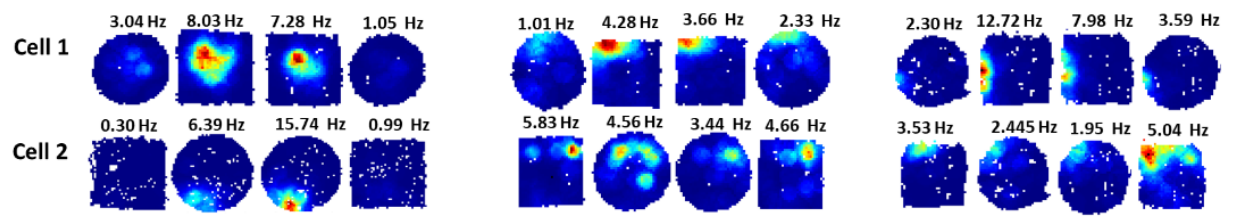

Al
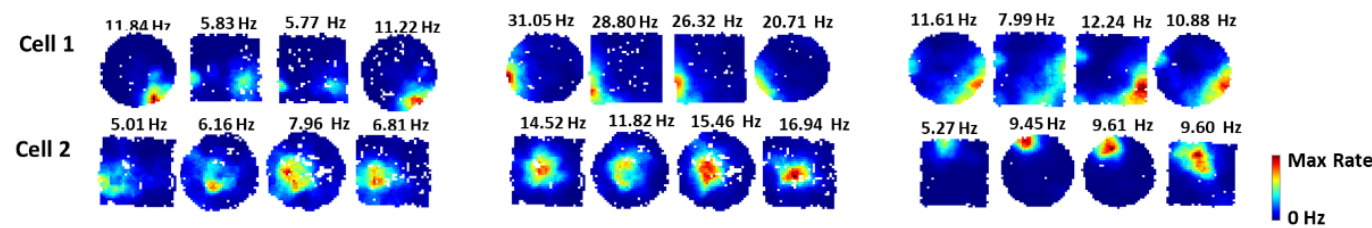

Figure 4. Representative rate maps for $Y, A U$ and $A l$ rats showing different degrees of remapping in the two-shape experiment. (A) Schematics showing the two-shape experiment. The sequence of order was either C-S-S-C or S-C-C-S. (B) Rate maps of two example cells from each age group in each CA3 subregion. Cells in $Y$ rats showed remapping between the two shapes in proximal and intermediate CA3 but fired in both shapes in distal CA3. Cells in AU rats showed remapping in proximal CA3, but fired in both shapes in intermediate and distal CA3. Cells in Al rats fired in both shapes in all CA3 subregions. These example cells have spatial correlation values between the different shapes that are within the second quartile (25-50\%) or the third quartile (50-75\%) from the population distribution.

To quantitatively assess the degree of remapping between the two shapes, we calculated population vector (PV) correlations (Fig. 5A), which are sensitive to the distribution of changes in the cells' firing locations and firing rates (i.e., global and rate remapping; (Leutgeb et al., 2005). In all age groups, population vectors in the same shape configurations (Fig. 5B, solid lines) were more strongly correlated than the population vectors in the different shape 
configurations (dotted lines). However, there were noticeable differences in the magnitude of PV correlation strengths between the shape configurations. In $\mathrm{Y}$ rats, the change in PV correlations in the same shape versus the different shape was greater in proximal and intermediate CA3 than in distal CA3. In AU rats, the change in PV correlations was greater in proximal CA3 than in intermediate and distal CA3. In Al rats, the change in PV correlations was small in all CA3 subregions.

To measure the change in PV correlation strengths between the same shapes and the different shapes, the PV correlation change was calculated. First, we summed the PV correlations for each of the bins (i.e., each of the values in the cumulative distribution plots in Fig. 5B). We then subtracted the summed PV correlation for the different shapes from the summed PV correlation for the same shapes, and divided this difference by the sum of the two quantities. This measure was calculated for each age group in each region. The statistical significance was calculated by a shuffling procedure in which the cells from both age groups under comparison were shuffled and randomly reassigned to each age group. The PV correlation difference was calculated from the shuffled data set, and this process was repeated 1000 times. The PV correlation change was significantly different between $\mathrm{Y}$ and $\mathrm{Al}$ rats and between $A U$ and $A I$ rats in proximal $C A 3$ but not between $Y$ and $A U$ rats (Fig. $5 C$ ), suggesting that $\mathrm{Y}$ and $\mathrm{AU}$ rats show greater remapping (i.e., greater change in $\mathrm{PV}$ correlation strengths) in the different shape configuration than Al rats. In intermediate CA3, the PV correlation change was significantly different between $\mathrm{Y}$ and $\mathrm{Al}$ and between $\mathrm{Y}$ and $\mathrm{AU}$ rats but not different between $A U$ and $A I$ rats, suggesting that $Y$ rats show greater remapping in the different shape configuration than $\mathrm{AU}$ and $\mathrm{Al}$ rats. In distal CA3, there were no significant differences between the age groups, suggesting that all age groups show similar remapping in the different shape configurations.

These results suggest that, similar to the cue-mismatch experiment, Al rats showed abnormally low remapping in proximal CA3 compared with $\mathrm{Y}$ and $\mathrm{AU}$ rats, but they were more 
similar to $Y$ and $A U$ rats in distal CA3. Similar to Figure 3D, to examine whether such an interaction between age groups and CA3 subregions was significant, the PV correlation change between age groups from one subregion was compared to another subregion (Fig. 5D).

Numerous (5/9) interactions between age groups and proximal-intermediate and intermediatedistal showed low $p$ values $(p<0.05)$, although they were not significant at $p=0.005$ level with Bonferroni correction. The data suggest that $A I$ rats remapped less than $Y$ and $A U$ rats in proximal CA3, whereas the amount of remapping in distal CA3 was similar across all 3 age groups. Moreover, compared to $\mathrm{Y}$ rats, AU rats appeared to show an abnormally low amount of remapping in intermediate $\mathrm{CA} 3$, similar to Al rats, which was not apparent in the cue-mismatch experiment (see also Supp. Fig S7). 
A)
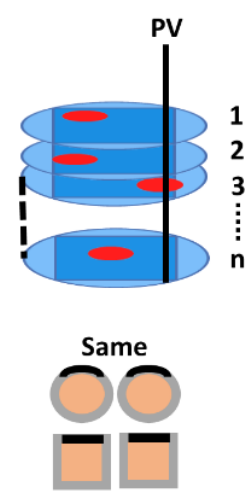

Different
B)
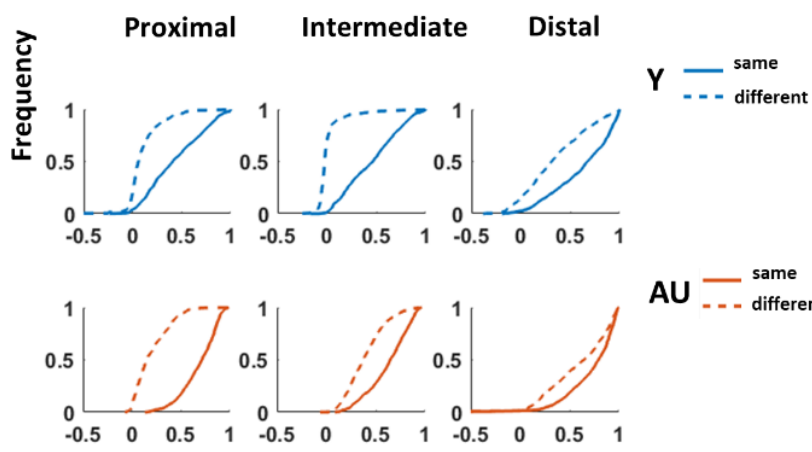

Al - same

AU - same

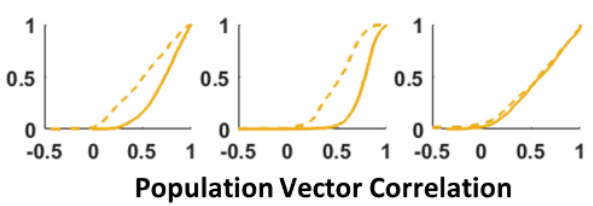

C)

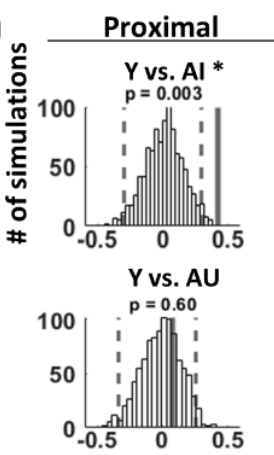

Intermediate Distal

D)

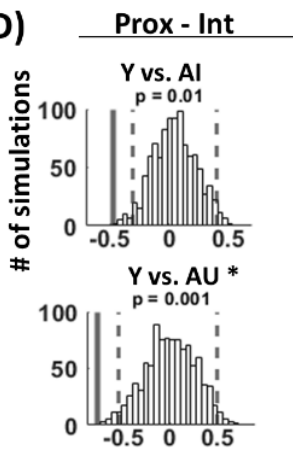

Int - Dist Prox - Dist
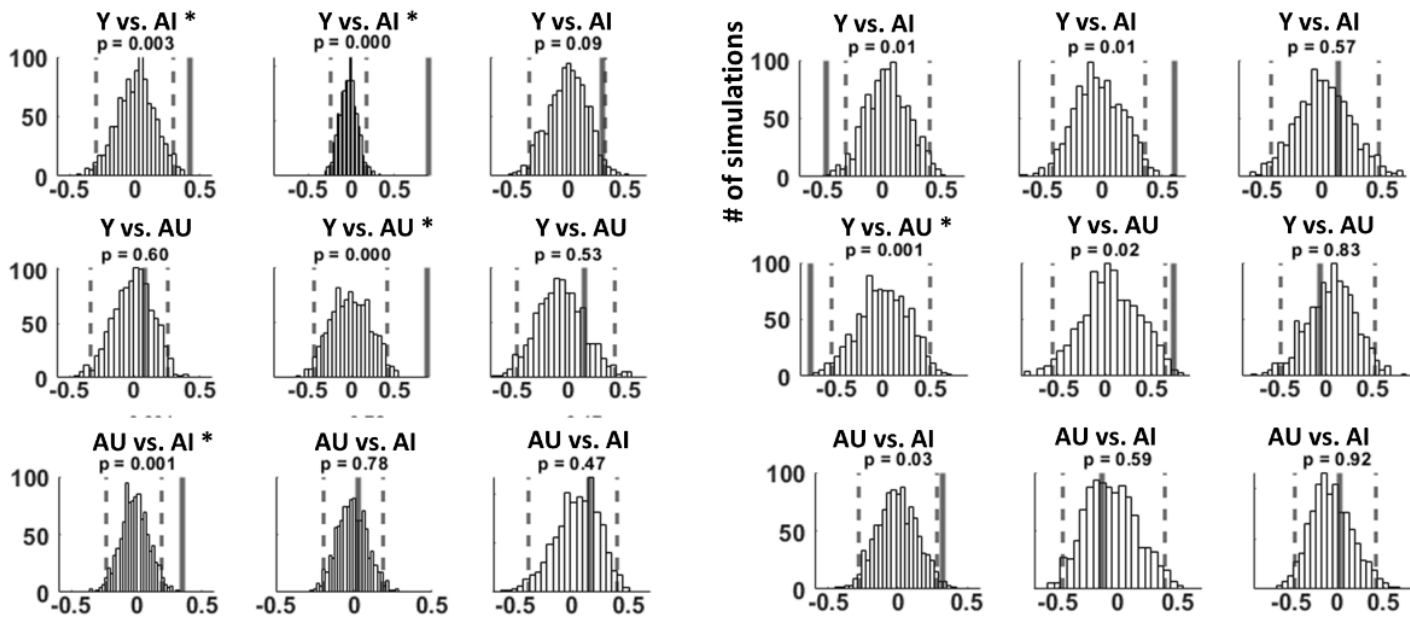

Normalized Sum Correlation Difference

Normalized Sum Correlation Difference

Figure 5. Representations for the different shape environments are less distinguishable in $A I$ rats compared to $Y$ and $A U$ rats. (A) Illustration showing the population vector correlation analysis (top). The rate maps of all CA3 cells were stacked, and the firing rates along the $z$ axis represent the population vector (PV) for each $x-y$ bin that was shared between the circle and the square. (Unshared bins between the two shapes were excluded). Correlations between the population vectors were computed between the same shapes (C-C or S-S) and the different shapes (C-S). (B) Cumulative distribution plots for PV correlations between the same shape (solid lines) and the different shape (dashed lines) in $Y$ (top row), AU (middle row), and Al rats (bottom row). PV correlation of 0 indicates strong remapping and $\mathrm{PV}$ correlation of 1 indicates completely correlated firing patterns between the two environments. (C-D) Shuffling analyses comparing the normalized sum PV correlation differences between the age groups in each CA3 subregion (C) and between the subregions (D). * denotes significance at $p=0.005$, Bonferroni corrected for 9 comparisons. 
Responses in the two-room experiment

For the two-room experiment, after session 4 in room A, the same rats from the twoshape experiment were taken to room B and were given additional sessions (sessions 5 and 6) in the square and the circle. All comparisons were made between session 4 in room A (last session in room A) and session 5 or 6 in room B (whichever session matched the same shape as session 4 in room A). Example rate maps showed that place fields changed their firing locations between the two rooms, or were active in only one room, in all CA3 subregions in $\mathrm{Y}$, $\mathrm{AU}$, and $\mathrm{Al}$ rats (Fig. 6A). Population vector correlations were very low (Fig. 6B) in all CA3 subregions for all age groups, and shuffling analysis did not show any difference between the age group comparisons in all CA3 subregions (Fig. 6C). These data suggest that when spatial location changes between the environments and the two environmental features are distinct, all age groups are able to dissociate the different rooms by remapping the place fields in all CA3 subregions. 
A)
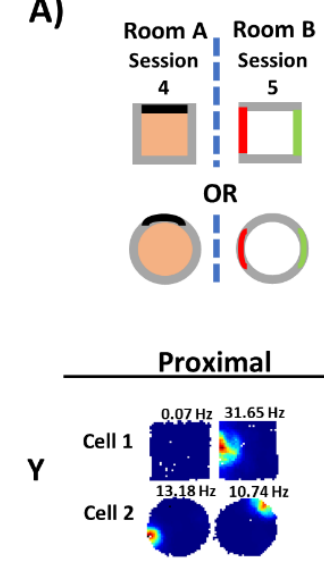

Intermediate

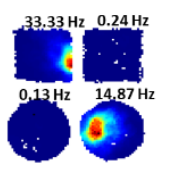

AU
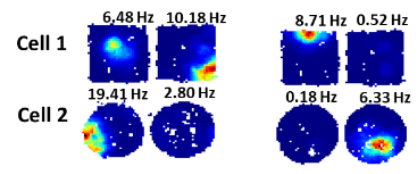

Al
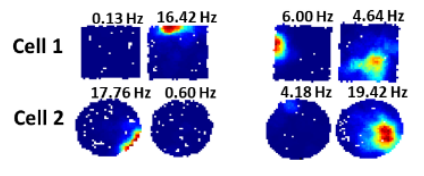

Distal
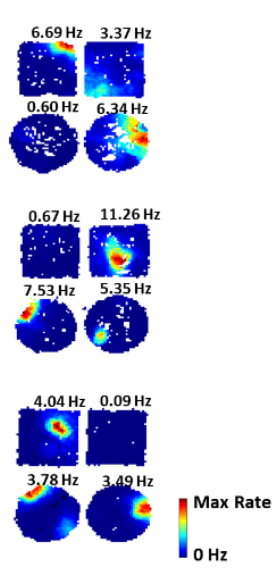

B)

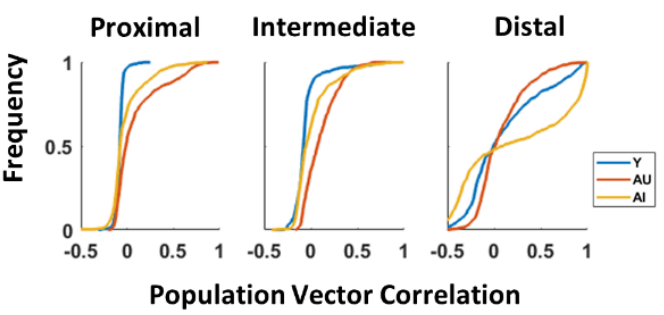

C)

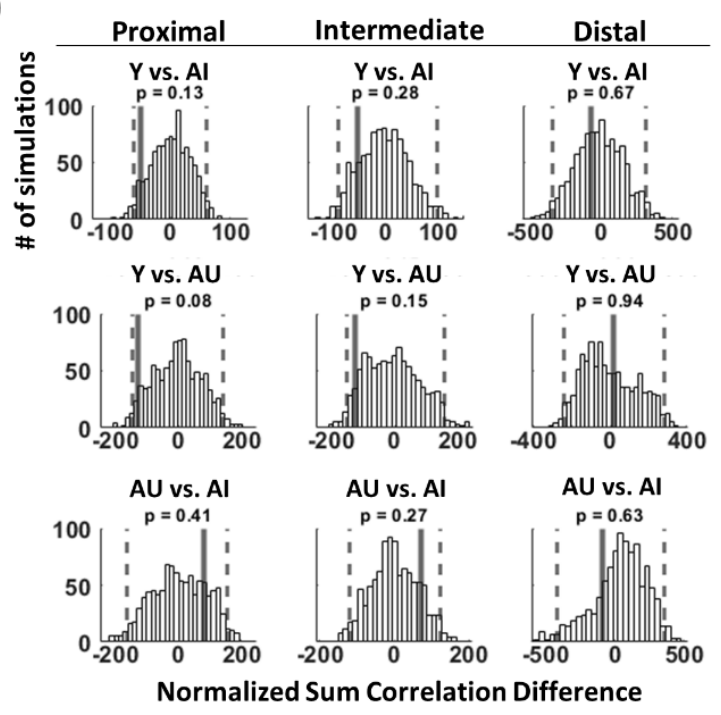

Figure 6. CA3 place cells in all age groups remap between the two rooms. (A) Schematics showing the two-room task (left). Rate maps of two example cells from each animal group in each CA3 subregion. Cells show remapping between the two rooms in all CA3 subregions in all age groups. (B) Cumulative distribution plots for PV correlation between Room A and Room B in each CA3 subregion. (C) Shuffling analysis comparing the normalized sum PV correlation differences between the animal groups in each CA3 subregion. None of the age comparisons are statistically different for any CA3 region.

\section{Discussion}

The current study showed a shift from pattern separation to pattern completion along the CA3 transverse axis in $\mathrm{Y}$ and $\mathrm{AU}$ rats that was not observed in Al rats (Fig. 7A). In the cuemismatch experiment, $\mathrm{Y}$ and $\mathrm{AU}$ rats showed a similar trend of a gradual decrease in remapping from proximal to distal CA3, while Al rats showed the opposite trend of an increase in remapping from proximal to distal CA3. In the two-shape experiment, there was an abrupt 
transition from weaker correlation in proximal and intermediate to stronger correlation in distal $\mathrm{CA} 3$ in $\mathrm{Y}$ rats, while the transition occurred between proximal and intermediate regions in $\mathrm{AU}$ rats. Although the transition point along the transverse axis was different between $\mathrm{Y}$ and $\mathrm{AU}$ rats, there was a shift from weaker correlation in proximal to stronger correlation in distal CA3 in both $\mathrm{Y}$ and $\mathrm{AU}$ rats. In contrast, there was strong correlation in all CA3 subregions in AI rats. Results from these two experiments suggest a bias toward pattern separation in proximal CA3 (i.e., more remapping) and a bias toward pattern completion in distal CA3 (i.e., less remapping) in $Y$ and $A U$ rats, showing a functional transition along the CA3 transverse axis. This functional shift was not evident in Al rats, as the results suggest pattern completion in all CA3 subregions in Al rats. In contrast, in the two-room experiment, there was remapping in all CA3 subregions in all age groups, suggesting that when the two environments do not share overlapping features and are in distinctly different locations (i.e., two different rooms), CA3 cells in all subregions discriminate between the two environments regardless of age and cognitive status. In agreement with prior studies on age-related hyperactivity of CA3 cells (H. Lee et al., 2021), these data suggest that proximal CA3 is a hub of age-related memory dysfunction in the hippocampal circuitry.

Although the transition patterns observed in the cue-mismatch and the two-shape experiments were somewhat different (Fig. 7A), this difference is consistent with two prior studies that showed that remapping along the CA3 transverse axis is nonlinear in $\mathrm{Y}$ rats $(\mathrm{H}$. Lee et al., 2015; Lu et al., 2015). Using the same cue-mismatch experiment, the Lee et al. (2015) study showed that, compared among each other, proximal CA3 showed relatively decorrelated representations but intermediate and distal CA3 showed relatively correlated representations, suggesting that the transition from pattern separation to pattern completion occurs between the proximal and intermediate regions. In contrast, Lu et al. (2015) showed an abrupt transition in remapping in an open-field, 2-room foraging task, with the representations of the two rooms showing dissimilarity in proximal and intermediate CA3 and similarity in distal CA3. While 
gradual or abrupt transitions from dissimilar to similar representations along the CA3 transverse axis may vary depending on the task and the degree to which the afferent representations differ, both studies consistently showed that the proximal region supports pattern separation and the distal region supports pattern completion. Similarly, in the current study, while the transition point differed between $\mathrm{Y}$ and $\mathrm{AU}$ rats in the cue-mismatch and the two-shape experiments (Fig. 7A), $Y$ and $A U$ rats showed pattern separation (strong remapping) in proximal CA3 and pattern completion (less remapping) in distal CA3, while Al rats showed pattern completion (less remapping) in proximal CA3, with a potential shift to more remapping in distal CA3.

Prior aging studies suggested that enhanced pattern completion might be favored in aged rats as the result of the hyperactivity in the pattern completion network of CA3 (Wilson et al., 2005, 2006). Based on the functional dissociation along the CA3 transverse axis, the simple logic is that hyperactivity in aged rats should be observed in distal CA3, which supports pattern completion. However, distal CA3 does not show hyperactivity in aged rats, and may even show hypoactivity. Instead, proximal CA3 shows hyperactivity (H. Lee et al., 2021). Since proximal CA3 supports pattern separation ( $\mathrm{H}$. Lee et al., 2015; Lu et al., 2015), how does hyperactive proximal CA3 favor pattern completion instead of pattern separation in aging? To resolve this apparent paradox, we previously proposed a model (Fig. 7B) of how age-related changes in CA3 firing rates along the transverse axis affect the attractor dynamics of CA3 $(\mathrm{H}$. Lee et al., 2021). We proposed that because proximal CA3 in aged rats presumably receives disrupted pattern-separated outputs from DG, the recurrent collaterals in proximal CA3, facilitated by the hyperactivity, may override the DG inputs to produce a single attractor basin that results in pattern completion. The possible hypoactivity in distal CA3 may result in weaker attractor basins in aged rats to result in bistable representations. The current study is consistent with the model by providing direct physiological evidence that representations in Al rats show abnormally high pattern completion in proximal CA3, with a potential weakening of pattern completion in distal CA3. 

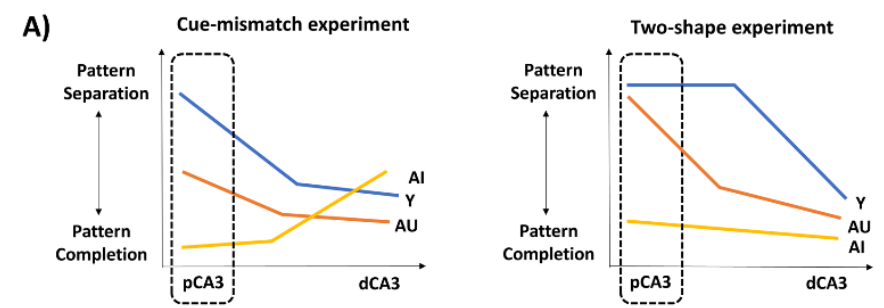

B)
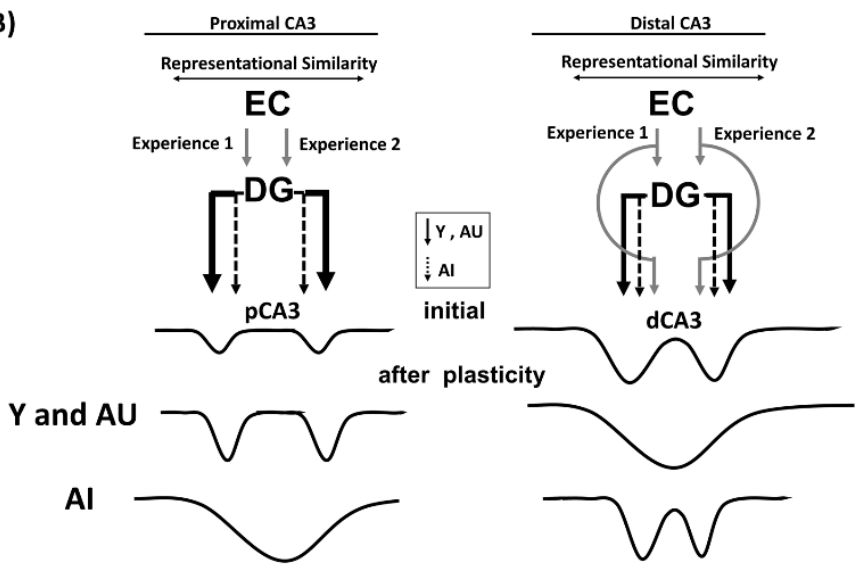

Figure 7. Hypothesized model of how the attractor dynamics of CA3 is affected by aging. (A) Summarized results from the two remapping experiments. $Y$ and $A U$ rats show a functional gradient [from pattern separation in proximal CA3 (pCA3) to pattern completion in distal CA3 (dCA3)] while Al rats show pattern completion in pCA3 and dCA3, with a potential weakening of pattern completion in dCA3. (B) In proximal CA3 (left), the EC neural representations of similar experiences (shown as distance between two input arrows along a "representational similarity" axis) will be pattern separated in the DG (indicated by the larger distance between the DG output to CA3 than the distance between the two EC inputs shown in thick, black solid lines). Proximal CA3, which receives stronger inputs from the DG (both upper and lower blades) coupled with minimal EC inputs, imposes the separated patterns on the CA3 attractor network. Through learning, the recurrent collaterals of pCA3 increases the strength of the attractor basins, resulting in pattern separation in pCA3 for $Y$ and AU rats. In contrast, for Al rats, dysfunction of the EC inputs and the local inhibitory circuitry of the DG hilus are hypothesized to reduce the ability of the DG to separate the similar EC input patterns (indicated by the close distance between the DG outputs to CA3 shown in black, broken lines). The hyperactivity of pCA3 cells in Al rats may further increase the relative strength of the pCA3 to override the DG inputs and cause a single basin of attraction, resulting in pattern completion in pCA3. In distal CA3 (right), there are weaker DG inputs (black solid lines) and stronger EC inputs (gray lines) compared to pCA3. The combined DG and EC inputs may impose initial patterns on dCA3 that are somewhat closer together and overlapping than those imposed in $\mathrm{PCA} 3$, and the stronger recurrent collateral system of dCA3 may merge the two attractor basins for a broader and deeper basin, resulting in pattern completion for $Y$ and $A U$ rats. In Al rats, weaker DG inputs (black, broken lines) and the possible hypoactivity of dCA3 cells may result in weaker attractor basins in $\mathrm{dCA} 3$ and prevent the merging of the attractor basins, resulting in two attractor states with relatively low energy barriers between them. (Part b adapted from Lee et al., 2021). 
Implications for aging

What are the implications of age-related deficits in proximal CA3? Previous studies have argued that proximal CA3 should be considered more as a functional component of the DG pattern-separation circuit than as a functional component of the distal CA3 pattern completion circuit (Hunsaker et al., 2008; H. Lee et al., 2015, 2020, 2021). The outputs from proximal and distal CA3 are topographically organized along the CA1 transverse axis, forming parallel processing streams along the hippocampal transverse axis (Fig. 8). Pattern-separated output from proximal CA3 and egocentric sensory information from lateral entorhinal cortex (LEC) target distal CA1 while pattern-completed output from distal CA3 and allocentric spatial information from medial entorhinal cortex (MEC) target proximal CA1 (Amaral \& Witter, 1989; Ishizuka et al., 1995; H. Lee et al., 2020). In aging, there is compelling evidence that the LEC is selectively vulnerable, including evidence of neurofibrillary tangles in the LEC earlier than the MEC in human aging (Braak \& Braak, 1995); hypometabolism in the LEC, but not in the MEC, in patients with mild cognitive impairment (Khan et al., 2014); and significantly reduced expression of reelin, a protein involved in cell migration and synaptic plasticity, in layer II of LEC, but not in MEC, of Al rats compared to $\mathrm{Y}$ and $\mathrm{AU}$ rats (Stranahan, Haberman, et al., 2011; Stranahan, Salas-Vega, et al., 2011). Profound impairment in the pattern separating function of the DG/proximal CA3 and the functions of the LEC suggest that the pCA3-dCA1-LEC pathway is more vulnerable in aging than the dCA3-pCA1-MEC pathway. MEC appears to be more associated with path integration and the creation of allocentric maps (the spatial context of experience), whereas LEC appears to be more associated with egocentric representations of the specific content (and perhaps temporal context; (Tsao et al., 2018)) of an experience (Knierim, 2015). Distal CA1, with its specific, reciprocal connectivity with LEC, appears to require input from proximal CA3 that is biased toward pattern separation to perform its function, whereas proximal CA1, with its specific, reciprocal connectivity with MEC, appears to require input from distal CA3 that is biased toward pattern completion. It is not known why these two 
processing streams require differential balances between pattern completion and pattern separation from CA3, but it is possible that the MEC-related pathway is biased toward pattern completing small changes to an environment, in order to retrieve the appropriate spatial context, and the LEC-related pathway is biased toward pattern separating representations of the specific items that were altered, in order to encode similar events that occur within the same context. In this scenario, the mnemonic deficits associated with aging arise more from dysfunction in the pattern separation processing of the individual items of experience, rather than the ability to correct for small errors or degraded inputs that arise from small changes to the representations of the allocentric, spatial contexts within which experiences occur.

Consistent with aged animal models, fMRI studies in aged individuals with deficits in lure discrimination tasks show heightened activity in DG/CA3 (Bakker et al., 2012, 2015; Reagh et al., 2018; Yassa et al., 2011). While it is difficult in human fMRI studies to distinguish DG BOLD signals from CA3 signals, it is possible that the DG/CA3 heightened activity in BOLD signals may largely reflect the heightened activity in proximal CA3, as proximal CA3 appears to be disproportionately large in humans, with $\sim 75 \%$ of CA3 apparently homologous to rodent proximal CA3 (Lim et al., 1997). Accordingly, the proportional increase in the size of proximal CA3 from rodents to primates suggests that adaptive pressures to support human episodic memory increased the relative role of the pCA3-dCA1-LEC pathway compared to the dCA3pCA1-MEC pathway, and disruption to the former pathway results in the age-related deficits in episodic memory. 


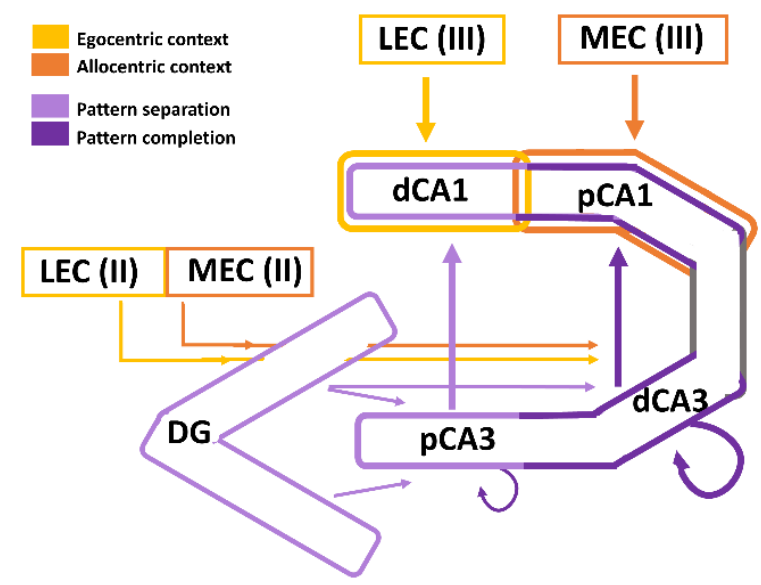

Figure 8. Parallel processing streams within the hippocampus. Allocentric contextual information (dark orange) from MEC projects to distal CA1 (dCA1) and egocentric content information (yellow) from LEC projects to proximal CA1 (pCA1). Both MEC and LEC directly project to the DG as well as to CA3, with stronger projection to distal CA3 (dCA3). The DG projects to CA3, with stronger projection to proximal CA3 (pCA3). The DG and pCA3, involved in pattern separation, are marked in light purple, and dCA3, with its strong recurrent circuitry, involved in pattern completion is marked in dark purple. Pattern-separated outputs from pCA3 preferentially project to dCA1, while pattern-completed outputs from dCA3 preferentially project to pCA1.

Remapping in the two-room

In the current study, Al rats showed place field rigidity in CA3 when changes were made by cue manipulations or different shape enclosures placed in the same location, which is consistent with prior aging studies showing CA3 place field rigidity in novel environments located in the same location (Robitsek et al., 2015; Wilson et al., 2005). However, in the tworoom experiment, in which there was an actual location change, Al rats showed remapping similar to $\mathrm{Y}$ and $\mathrm{AU}$ rats. A possible explanation for the remapping observed in $\mathrm{Al}$ rats is that the two rooms were different enough that transformation of entorhinal input patterns was not necessary (Knierim \& Neunuebel, 2016). The dynamic switch from pattern separation to pattern completion in CA3 has been modeled as a sigmoidal function (Guzowski et al., 2004; McClelland \& Goddard, 1996). When changes in inputs are small, CA3 performs pattern completion resulting in output representations that are similar. When changes in inputs are 
large, CA3 performs pattern separation resulting in output representations that are dissimilar. It has been hypothesized that in aged rats, the sigmoidal input-output function is skewed towards pattern completion and requires greater changes in inputs to generate output patterns that are dissimilar (Wilson et al., 2006). Similarly, aged individuals showed that larger changes in inputs are required to successfully separate lure items from target items (Yassa et al., 2011), suggesting that more dissimilarity in input patterns is necessary for successful discrimination. Thus, the changes in inputs between the two rooms may have been large enough to result in dissimilar output representations in Al rats.

\section{Cognitive resilience in $A U$ rats}

While memory function is susceptible to aging, a subpopulation of aged animals (including aged humans) is able to retain normal memory function. We previously showed that hyperactivity localized to proximal CA3 region was present in both AU and Al rats $(\mathrm{H}$. Lee et al., 2021). Yet, $A U$ rats showed a transition from pattern separation to pattern completion in CA3 but $\mathrm{Al}$ rats showed pattern completion in all regions of CA3. While AU rats did not always show statistical difference from Al rats, the trends observed in $A U$ rats were more similar to $Y$ rats than $\mathrm{Al}$ rats. What makes $\mathrm{AU}$ rats more resilient to such age-related cognitive impairment? There are subtle changes in hippocampal circuitry that may account for possible compensatory mechanisms in AU rats. Postsynaptic inhibition is enhanced in granule cells in AU rats compared to Al rats (Tran et al., 2018, 2019), and somatostatin-positive HIPP interneurons are intact in AU rats but reduced in Al rats (Spiegel et al., 2013). Enhanced inhibition in granule cells in AU rats may support sparse coding in the DG, which is essential for pattern separation. There is also a redistribution of synaptic weights between age groups, where AMPAR expression in stratum lucidum (the layer where mossy fibers make synaptic contacts with CA3) is higher in AU rats but AMPAR expression is significantly higher in stratum radiatum (the layer where CA3 makes autoassociational connections) in Al rats (Buss et al., 2021). Enhanced 
AMPAR expression in stratum lucidum may support increased feedforward activation of pattern separated outputs to CA3. However, exactly how these changes provide compensatory mechanisms in AU rats still remain largely unknown and need further study.

\section{Summary}

The present study demonstrates that age-related impairments in pattern separation is localized preferentially to proximal CA3 in Al rats. When changes are made to the environment, neural representations in $\mathrm{Y}$ rats are more orthogonal in proximal CA3 than in distal CA3, suggesting a functional gradient (pattern separation to pattern completion) along the CA3 transverse axis. In contrast, Al rats show less orthogonal representations in proximal CA3 compared to $\mathrm{Y}$ rats but more generalized representations in distal CA3 that are similar to $\mathrm{Y}$ rats. Thus, enhanced pattern completion in Al rats may result from proximal CA3 that is more biased towards pattern completion rather than pattern separation. Furthermore, while AU rats show similar trends as $\mathrm{Y}$ rats, the magnitude of orthogonalization in proximal CA3 is not always similar to $Y$ rats, suggesting that $A U$ rats are not equivalent to $Y$ rats.

\section{Methods}

\section{Subjects and Surgery}

Male Long-Evans rats (retired breeders at 9 months) were obtained from Charles River Laboratories (Raleigh, NC) and housed in a vivarium at Johns Hopkins University until age 2226 months. Young rats (3-6 months) were also obtained from Charles River Laboratories. Prior to the onset of water maze behavioral testing, rats were individually housed with ad libitum access to food and water during a 12-hour light/dark cycle. After the water maze testing, rats underwent hyperdrive implant surgery and were housed during a reversed 12-hour light/dark cycle. All recordings were done in the dark cycle. All animal care, housing, and surgical 
procedures conformed to the National Institute of Health standards using protocols approved by the Institutional Animal Care and Use Committee at Johns Hopkins University.

All aged rats were prescreened for spatial learning ability in the Morris water maze as previously described (Lee et al., 2021) prior to the implantation of recording electrodes. Four of the 8 young rats also pretested in the water maze. The remaining 4 young rats were from a previous study (H. Lee et al., 2015) and were not tested in the Morris water maze. Thus, in total, 14 aged rats were tested at 22-24 months and 4 young rats were tested at 3-4 months in the water maze. These rats were trained for 8 days ( 3 trials per day) to locate a submerged escape platform that remained at the same location in the water maze tank, with every sixth trial as a probe trial (no escape platform for the first 30 seconds). The learning index (LI), an average of weighted search proximity scores obtained during probe trials (Gallagher et al., 1993), was used to classify animals as aged-impaired ( $\mathrm{Al}$; $\mathrm{LI}>240$ ) or aged-unimpaired (AU; LI < 240), using an a priori LI criterion from previous studies (Branch et al., 2019; Gallagher et al., 1993). On day 9 , rats were given six trials to locate a visible platform above the water level to screen for nonspecific task impairment such as swimming behavior and escape motivation. Following water maze testing, rats were placed on restricted food access to reduce their body weight to $85 \%$ while they were given foraging sessions (20 mins per day for 10 days) in a cylindrical apparatus to accustom the rats to forage for chocolate pellets (Bio-Serv, Flemington, $\mathrm{NJ}$ ) before the subsequent training on the circular track.

A custom-built recording hyperdrive that contained 15 independently moveable tetrodes was surgically implanted over the right hemisphere. To optimize the drive placement, recordings were performed during the surgery to find the lateral edge of CA3. The most lateral tetrode placement ranged from 3.7 to $4.2 \mathrm{~mm}$ lateral to bregma and from 3.4 to 4.0 posterior to bregma. The tetrode array was configured as an oval bundle, approximately $1.2 \mathrm{~mm}$ in length and $0.9 \mathrm{~mm}$ in width, with a $3 \times 6$ array of tetrodes spaced approximately $300 \mu \mathrm{m}$ apart. The 
array was implanted at an angle of $\sim 35^{\circ}$, which is approximately parallel to the transverse axis of the dorsal hippocampus.

Local-global cue-conflict manipulation

All rats were trained to run in a clockwise direction on a circular track for randomly placed chocolate pellets for 2-3 weeks while the tetrodes were being lowered to CA3. The circular track (76 cm outer diameter, $56 \mathrm{~cm}$ inner diameter) with salient local texture cues was placed in a black-curtained room with salient global cues. The cue-conflict manipulation experiment was conducted for 4 days, with 5 sessions each day. The rat was disoriented between each session. Three standard (STD) sessions (local and global cue relationships remained constant) were interleaved with two mismatch (MIS) sessions (local and global cues were rotated by equal increments but in opposite directions, producing mismatch angles of $45^{\circ}$, $90^{\circ}, 135^{\circ}$, and $\left.180^{\circ}\right)$. Mismatch angles were chosen in pseudorandom order such that each angle was chosen once during the first 2 days of recording and once again during the second 2 days. On each recording day, baseline sessions ( $15 \mathrm{~min})$ in which the rat rested in the holding dish were recorded prior to the start and at the end of the experiment, in order to compare recording stability before and after the experiment. Because $45^{\circ}$ mismatch is a minor degree of separation and the cell responses in $45^{\circ}$ MIS session do not differ markedly from STD sessions (see Lee et al., 2015), cells recorded during $45^{\circ}$ MIS sessions were not included in this analysis.

\section{Two-shape experiment}

During the 4 days of the cue-conflict manipulation task, subsets of the rats ( $3 \mathrm{Y}, 6 \mathrm{AU}, 6$ $\mathrm{Al})$ were trained in the two shapes [square $(67 \mathrm{~cm} \times 67 \mathrm{~cm})$ and circle $(76 \mathrm{~cm}$ diameter)] placed at a fixed location in room A. On day 1 , rats were trained to forage for chocolate pellets for 20 min once in the circle chamber and once in the square chamber. On days 2 - 4, rats were trained for 10 min each session in the square and the circle for a total of 4 sessions in a random order ( 2 sessions in each shape). A striped cue card was placed on the north wall inside the 
chambers, and new brown paper floor was replaced between each session. The rat was allowed to rest for 5 - 10 min on the pedestal between each session. After 4 days of training, the two-shape experiment was conducted for 2 days, 4 sessions each day. During the 2 recording days, the order of the shapes was circle-box-box-circle and box-circle-circle-box, although which order was given on the first day was random.

Two-room experiment

After 4 sessions of the different shape experiment on each recording day, the rat was placed on a pedestal and taken to room B. Two additional 10 min sessions were given in room $\mathrm{B}$, once in the square chamber and once in the circle chamber. Two new cue cards (brown cue card on the west wall and gray hexagon shaped cue card on the east wall) were placed inside the chambers, and a new white paper floor was replaced between each session. The order of the square and circular shape was random. There was no prior training in room B.

\section{Electrophysiological Recordings}

Tetrodes were made from $17-\mu \mathrm{m}$ platinum-iridium wires (California Fine Wire Co., Grover Beach, CA), with impedance reduced to $\sim 120$ kOhms by electroplating with platinum black. Neural signals were recorded using a 64-channel wireless transmitter system (Triangle Biosystems International, Durham, NC) and transmitted to a Cheetah Data Acquisition System (Neuralynx, Bozeman, MT). The signals were amplified 1,000-5,000 times and filtered between $600 \mathrm{~Hz}$ and $6 \mathrm{kHz}$ (for units) or 1 and $600 \mathrm{~Hz}$ for local field potentials (LFPs). The spike waveforms above a threshold of $40-70 \mu \mathrm{V}$ were sampled for $1 \mathrm{~ms}$ at $32 \mathrm{kHz}$, and LFPs were continuously sampled at $32 \mathrm{kHz}$. The rat's position was tracked with an overhead camera recording light emitting diodes (LEDs) position over the head of the rat (red LEDs in front and green LEDs behind) at $30 \mathrm{~Hz}$.

Data Analysis

Unit Isolation 
Multiple waveform characteristics (e.g., spike amplitude and energy) were used to isolate single units using custom-written manual cluster-cutting software. Cells were assigned to subjective isolation quality scores from 1 (very good) to 5 (poor), depending on the cluster separation from the other clusters and from the background activity level. The isolation quality scores were made completely independently of any behavioral correlates of the cells. Using a K-means clustering analysis on three parameters (spike width, mean firing rate, and burst index), cells were classified as putative pyramidal cells (low-rate broad spikes, bursty) or putative interneurons (high-rate, narrow spikes, nonbursty) for each session (Lee et al., 2021). If a cell was classified as a putative interneuron (i.e., a high rate cell) in one of the sessions, that cell was classified as a putative interneuron for all sessions. Only well-isolated putative pyramidal cells (with isolation quality scores 1-3) were included in the analysis. Putative interneurons were excluded from the analysis.

\section{Rate Maps and Place Fields}

The position and the head direction of the rat were based on tracking the LEDs on the headstage connected to the hyperdrive. Analysis was performed on data restricted to times when the animal was moving forward at a speed $>3 \mathrm{~cm} / \mathrm{s}$. For $2 \mathrm{D}$ rate maps, the $\mathrm{x}$ and $\mathrm{y}$ dimensions of the camera image (640 x 480 pixels) were divided into bin sizes of 10 pixels (2.5 $\mathrm{cm}$ square bins). To create a firing rate map of a cell, the number of spikes in each bin was divided by the amount of time the rat spent in that bin. Rate maps for the circle-square environments were smoothed using the adaptive binning method (Skaggs et al., 1996); rate maps for the circular track were smoothed with a Gaussian filter $\left(\sigma=3^{\circ}\right)$. For the cue-conflict manipulation data, analysis was performed on data restricted to times when the animal's head was within the boundaries of the circular track, and the circular $2 \mathrm{D}$ rate maps were transformed into linear rate maps by converting the rat's Cartesian position into units of degrees on the track. Linearized rate maps were divided into 360 bins ( $1 \%$ bin). Rate maps were used to calculate the spatial information score (Skaggs et al., 1996), the mean and the peak firing rates. Place cells 
were identified as neurons with spatial information scores $>0.75$ bit/spike, spatial information significance $p<0.01$, and number of velocity-filtered spikes $>50$. Spatial information significance was calculated with a shuffling procedure, in which the spike train and the position train were shifted relative to each other by a random time (minimum $30 \mathrm{~s}$ ), the rate map was recalculated, and a spatial information score was calculated. This procedure was performed 1,000 times, and the cell was considered significant at $p<0.01$ if the true information score exceeded the values of more than 990 scores from the shuffling procedure. The peak firing rate was the maximum firing rate in the rate map, and the mean firing rate was calculated by dividing the number of spikes by the duration of the session.

Rotational Analysis

The rotation angle of rate maps between the sessions was determined for each cell that passed the inclusion criteria in both the STD and the MIS sessions. The linearized rate map in the MIS session was circularly shifted in $1^{\circ}$ increments and correlated with the linearized rate map in the STD session. The angle producing the maximum correlation was assigned as that cell's rotation angle.

Population correlation matrices

Population correlation matrices were created by forming normalized firing rate vectors for the sample of cells at each $1^{\circ}$ bin of the track to create 360 firing rate vectors. The firing rate of each cell in each bin is normalized to that cell's peak firing rate. The correlation matrix contains the Pearson product moment correlations values for each of the $360 \times 360$ firing rate vectors. A band of high correlation along the $0^{\circ}$ main diagonal indicates that the representation maintained coherence and did not change between the two environments.

\section{Population Vector Correlation}

Rate maps for the entire population of cells were arranged in an $x-y-z$ stack, where $x$ and y represent the two spatial dimensions and $z$ represents the cell-identity index. The distribution of mean firing rates along the $z$ axis represent the composite population vector for a given $x-y$ 
location. Population vectors for corresponding positions were correlated across pairs of sessions. Nonoverlapping $x-y$ locations between the two shapes were excluded from the PV correlations.

Statistical Analysis

All statistical tests were calculated using Matlab (Mathworks, Natick, MA). Random shuffling test was used to determine the statistical significance, as described in the corresponding Results section. Statistical analysis was two-tailed and considered significant at $p<0.005$ with Bonferroni correction for 9 comparisons, unless stated otherwise.

\section{Histological Procedures}

Rats were deeply anesthetized and perfused with $4 \%$ formaldehyde. Frozen coronal sections $(40 \mu \mathrm{m})$ were cut and stained with cresyl violet. Images of the brain slices were acquired with an IC Capture DFK 41BU02 camera (The Imaging Source, Charlotte) attached to a Motic SMZ - 168 stereoscope. All tetrode tracks were identified, and the lowest point of the track was used to determine the recording position. Distance of the CA3 tetrode was measured manually from the proximal end and scaled by the total length of CA3. The normalized position of the tetrode along the CA3 transverse axis ranged from 0 to 1 , with the distal end as 1 .

\section{Acknowledgements}

We thank Robert McMahan, Andrew Sherwood, and Kimberly Knah for assistance in running the behavior experiments and histological procedures. We thank Audrey Branch, William Hockeimer, and Ravikrishnan Jayakumar for helpful discussion. Supported by grant P01 AG009973. 


\section{Disclosure}

M.G. is the founder of AgeneBio Incorporated, a biotechnology company that is dedicated to discovery and development of therapies to treat cognitive impairment. M.G. has a financial interest in the company and is an inventor on Johns Hopkins University's intellectual property that is licensed to AgeneBio. Otherwise, M.G. has had no consulting relationship with other public or private entities in the past three years and has no other financial holdings that could be perceived as constituting a potential conflict of interest. All conflicts of interest are managed by Johns Hopkins University. All other authors have nothing to disclose.

\section{Author Contributions}

H.L. and J.J.K. designed the experiments; H.L., A.T., N.L., collected the data; H.L. and Z.W. analyzed the data; J.J.K., S.Z., M.G. provided supervision; J.J.K. and M.G. provided funding; H.L. wrote the initial draft of the manuscript; H.L. and J.J.K. wrote the manuscript; all authors provided comments and feedback on the manuscript.

\section{References}

1. Amaral, D. G., \& Witter, M. P. (1989). The three-dimensional organization of the hippocampal formation: A review of anatomical data. Neuroscience, 31(3), 571-591. https://doi.org/10.1016/0306-4522(89)90424-7

2. Anderson, M. I., \& Jeffery, K. J. (2003). Heterogeneous modulation of place cell firing by changes in context. The Journal of Neuroscience: The Official Journal of the Society for Neuroscience, 23(26), 8827-8835.

3. Bakker, A., Albert, M. S., Krauss, G., Speck, C. L., \& Gallagher, M. (2015). Response of the medial temporal lobe network in amnestic mild cognitive impairment to therapeutic 
intervention assessed by fMRI and memory task performance. Neurolmage. Clinical, 7 , 688-698. https://doi.org/10.1016/j.nicl.2015.02.009

4. Bakker, A., Krauss, G. L., Albert, M. S., Speck, C. L., Jones, L. R., Stark, C. E., Yassa, M. A., Bassett, S. S., Shelton, A. L., \& Gallagher, M. (2012). Reduction of hippocampal hyperactivity improves cognition in amnestic mild cognitive impairment. Neuron, 74(3), 467474. https://doi.org/10.1016/j.neuron.2012.03.023

5. Braak, H., \& Braak, E. (1995). Staging of Alzheimer's disease-related neurofibrillary changes. Neurobiology of Aging, 16(3), 271-278; discussion 278-284. https://doi.org/10.1016/0197-4580(95)00021-6

6. Branch, A., Monasterio, A., Blair, G., Knierim, J. J., Gallagher, M., \& Haberman, R. P. (2019). Aged rats with preserved memory dynamically recruit hippocampal inhibition in a local/global cue mismatch environment. Neurobiology of Aging, 76, 151-161. https://doi.org/10.1016/j.neurobiolaging.2018.12.015

7. Buss, E. W., Corbett, N. J., Roberts, J. G., Ybarra, N., Musial, T. F., Simkin, D., MolinaCampos, E., Oh, K.-J., Nielsen, L. L., Ayala, G. D., Mullen, S. A., Farooqi, A. K., D'Souza, G. X., Hill, C. L., Bean, L. A., Rogalsky, A. E., Russo, M. L., Curlik, D. M., Antion, M. D., ... Nicholson, D. A. (2021). Cognitive aging is associated with redistribution of synaptic weights in the hippocampus. Proceedings of the National Academy of Sciences of the United States of America, 118(8), e1921481118. https://doi.org/10.1073/pnas.1921481118

8. Ebert, P. L., \& Anderson, N. D. (2009). Proactive and retroactive interference in young adults, healthy older adults, and older adults with amnestic mild cognitive impairment. Journal of the International Neuropsychological Society: JINS, 15(1), 83-93. https://doi.org/10.1017/S1355617708090115

9. Emery, L., Hale, S., \& Myerson, J. (2008). Age differences in proactive interference, working memory, and abstract reasoning. Psychology and Aging, 23(3), 634-645. https://doi.org/10.1037/a0012577

10. Ferbinteanu, J., \& Shapiro, M. L. (2003). Prospective and retrospective memory coding in the hippocampus. Neuron, 40(6), 1227-1239. https://doi.org/10.1016/s0896-6273(03)007529

11. Gallagher, M., Burwell, R., \& Burchinal, M. (1993). Severity of spatial learning impairment in aging: Development of a learning index for performance in the Morris water maze. Behavioral Neuroscience, 107(4), 618-626. https://doi.org/10.1037//0735-7044.107.4.618

12. Guzowski, J. F., Knierim, J. J., \& Moser, E. I. (2004). Ensemble dynamics of hippocampal regions CA3 and CA1. Neuron, 44(4), 581-584.

https://doi.org/10.1016/j.neuron.2004.11.003 
13. Haberman, R. P., Branch, A., \& Gallagher, M. (2017). Targeting Neural Hyperactivity as a Treatment to Stem Progression of Late-Onset Alzheimer's Disease. Neurotherapeutics: The Journal of the American Society for Experimental NeuroTherapeutics, 14(3), 662-676. https://doi.org/10.1007/s13311-017-0541-z

14. Hunsaker, M. R., Rosenberg, J. S., \& Kesner, R. P. (2008). The role of the dentate gyrus, CA3a,b, and CA3c for detecting spatial and environmental novelty. Hippocampus, 18(10), 1064-1073. https://doi.org/10.1002/hipo.20464

15. Ishizuka, N., Cowan, W. M., \& Amaral, D. G. (1995). A quantitative analysis of the dendritic organization of pyramidal cells in the rat hippocampus. The Journal of Comparative Neurology, 362(1), 17-45. https://doi.org/10.1002/cne.903620103

16. Ishizuka, N., Weber, J., \& Amaral, D. G. (1990). Organization of intrahippocampal projections originating from CA3 pyramidal cells in the rat. The Journal of Comparative Neurology, 295(4), 580-623. https://doi.org/10.1002/cne.902950407

17. Khan, U. A., Liu, L., Provenzano, F. A., Berman, D. E., Profaci, C. P., Sloan, R., Mayeux, R., Duff, K. E., \& Small, S. A. (2014). Molecular drivers and cortical spread of lateral entorhinal cortex dysfunction in preclinical Alzheimer's disease. Nature Neuroscience, 17(2), 304-311. https://doi.org/10.1038/nn.3606

18. Knierim, J. J. (2002). Dynamic interactions between local surface cues, distal landmarks, and intrinsic circuitry in hippocampal place cells. The Journal of Neuroscience: The Official Journal of the Society for Neuroscience, 22(14), 6254-6264. https://doi.org/20026608

19. Knierim, J. J. (2015). The hippocampus. Current Biology: CB, 25(23), R1116-1121. https://doi.org/10.1016/j.cub.2015.10.049

20. Knierim, J. J., \& Hamilton, D. A. (2011). Framing spatial cognition: Neural representations of proximal and distal frames of reference and their roles in navigation. Physiological Reviews, 91(4), 1245-1279. https://doi.org/10.1152/physrev.00021.2010

21. Knierim, J. J., \& Neunuebel, J. P. (2016). Tracking the flow of hippocampal computation: Pattern separation, pattern completion, and attractor dynamics. Neurobiology of Learning and Memory, 129, 38-49. https://doi.org/10.1016/j.nlm.2015.10.008

22. Koh, M. T., Haberman, R. P., Foti, S., McCown, T. J., \& Gallagher, M. (2010). Treatment strategies targeting excess hippocampal activity benefit aged rats with cognitive impairment. Neuropsychopharmacology: Official Publication of the American College of Neuropsychopharmacology, 35(4), 1016-1025. https://doi.org/10.1038/npp.2009.207 
23. Lacy, J. W., Yassa, M. A., Stark, S. M., Muftuler, L. T., \& Stark, C. E. L. (2011). Distinct pattern separation related transfer functions in human CA3/dentate and CA1 revealed using high-resolution fMRI and variable mnemonic similarity. Learning \& Memory, 18(1), 15-18. https://doi.org/10.1101/lm.1971111

24. Lee, H., GoodSmith, D., \& Knierim, J. J. (2020). Parallel processing streams in the hippocampus. Current Opinion in Neurobiology, 64, 127-134. https://doi.org/10.1016/j.conb.2020.03.004

25. Lee, H., Wang, C., Deshmukh, S. S., \& Knierim, J. J. (2015). Neural Population Evidence of Functional Heterogeneity along the CA3 Transverse Axis: Pattern Completion versus Pattern Separation. Neuron, 87(5), 1093-1105. https://doi.org/10.1016/j.neuron.2015.07.012

26. Lee, H., Wang, Z., Zeger, S. L., Gallagher, M., \& Knierim, J. J. (2021). Heterogeneity of AgeRelated Neural Hyperactivity along the CA3 Transverse Axis. The Journal of Neuroscience: The Official Journal of the Society for Neuroscience, 41(4), 663-673. https://doi.org/10.1523/JNEUROSCI.2405-20.2020

27. Lee, I., Yoganarasimha, D., Rao, G., \& Knierim, J. J. (2004). Comparison of population coherence of place cells in hippocampal subfields CA1 and CA3. Nature, 430(6998), 456459. https://doi.org/10.1038/nature02739

28. Leutgeb, S., Leutgeb, J. K., Barnes, C. A., Moser, E. I., McNaughton, B. L., \& Moser, M.-B. (2005). Independent codes for spatial and episodic memory in hippocampal neuronal ensembles. Science (New York, N.Y.), 309(5734), 619-623. https://doi.org/10.1126/science.1114037

29. Lim, C., Blume, H. W., Madsen, J. R., \& Saper, C. B. (1997). Connections of the hippocampal formation in humans: I. The mossy fiber pathway. The Journal of Comparative Neurology, 385(3), 325-351. https://doi.org/10.1002/(sici)10969861(19970901)385:3<325::aid-cne1>3.0.c0;2-5

30. Lu, L., Igarashi, K. M., Witter, M. P., Moser, E. I., \& Moser, M.-B. (2015). Topography of Place Maps along the CA3-to-CA2 Axis of the Hippocampus. Neuron, 87(5), 1078-1092. https://doi.org/10.1016/j.neuron.2015.07.007

31. Lustig, C., \& Hasher, L. (2001). Implicit memory is not immune to interference. Psychological Bulletin, 127(5), 618-628. https://doi.org/10.1037/0033-2909.127.5.618

32. Markus, E. J., Qin, Y. L., Leonard, B., Skaggs, W. E., McNaughton, B. L., \& Barnes, C. A. (1995). Interactions between location and task affect the spatial and directional firing of hippocampal neurons. The Journal of Neuroscience: The Official Journal of the Society for Neuroscience, 15(11), 7079-7094. 
33. Marr, D. (1971). Simple memory: A theory for archicortex. Philosophical Transactions of the Royal Society of London. Series B, Biological Sciences, 262(841), 23-81. https://doi.org/10.1098/rstb.1971.0078

34. Marrone, D. F., Satvat, E., Odintsova, I. V., \& Gheidi, A. (2014). Dissociation of spatial representations within hippocampal region CA3. Hippocampus, 24(12), 1417-1420. https://doi.org/10.1002/hipo.22367

35. Maurer, A. P., Johnson, S. A., Hernandez, A. R., Reasor, J., Cossio, D. M., Fertal, K. E., Mizell, J. M., Lubke, K. N., Clark, B. J., \& Burke, S. N. (2017). Age-related Changes in Lateral Entorhinal and CA3 Neuron Allocation Predict Poor Performance on Object Discrimination. Frontiers in Systems Neuroscience, 11, 49. https://doi.org/10.3389/fnsys.2017.00049

36. McClelland, J. L., \& Goddard, N. H. (1996). Considerations arising from a complementary learning systems perspective on hippocampus and neocortex. Hippocampus, 6(6), 654-665. https://doi.org/10.1002/(SICI)1098-1063(1996)6:6<654::AID-HIPO8>3.0.CO;2-G

37. McNaughton, B. L., \& Morris, R. G. M. (1987). Hippocampal synaptic enhancement and information storage within a distributed memory system. Trends in Neurosciences, 10(10), 408-415. https://doi.org/10.1016/0166-2236(87)90011-7

38. Moita, M. A. P., Rosis, S., Zhou, Y., LeDoux, J. E., \& Blair, H. T. (2004). Putting fear in its place: Remapping of hippocampal place cells during fear conditioning. The Journal of Neuroscience: The Official Journal of the Society for Neuroscience, 24(31), 7015-7023. https://doi.org/10.1523/JNEUROSCI.5492-03.2004

39. Muller, R. U., \& Kubie, J. L. (1987). The effects of changes in the environment on the spatial firing of hippocampal complex-spike cells. The Journal of Neuroscience: The Official Journal of the Society for Neuroscience, 7(7), 1951-1968.

40. Nakamura, N. H., Flasbeck, V., Maingret, N., Kitsukawa, T., \& Sauvage, M. M. (2013). Proximodistal segregation of nonspatial information in CA3: Preferential recruitment of a proximal CA3-distal CA1 network in nonspatial recognition memory. The Journal of Neuroscience: The Official Journal of the Society for Neuroscience, 33(28), 11506-11514. https://doi.org/10.1523/JNEUROSCI.4480-12.2013

41. Neunuebel, J. P., \& Knierim, J. J. (2014). CA3 retrieves coherent representations from degraded input: Direct evidence for CA3 pattern completion and dentate gyrus pattern separation. Neuron, 81(2), 416-427. https://doi.org/10.1016/j.neuron.2013.11.017

42. O'Keefe, J., \& Dostrovsky, J. (1971). The hippocampus as a spatial map. Preliminary evidence from unit activity in the freely-moving rat. Brain Research, 34(1), 171-175. https://doi.org/10.1016/0006-8993(71)90358-1 
43. O'Keefe, J., \& Nadel, L. (1978). The Hippocampus as a Cognitive Map. Oxford: Clarendon Press. https://repository.arizona.edu/handle/10150/620894

44. O'Reilly, R. C., \& McClelland, J. L. (1994). Hippocampal conjunctive encoding, storage, and recall: Avoiding a trade-off. Hippocampus, 4(6), 661-682.

https://doi.org/10.1002/hipo.450040605

45. Reagh, Z. M., Noche, J. A., Tustison, N. J., Delisle, D., Murray, E. A., \& Yassa, M. A. (2018). Functional Imbalance of Anterolateral Entorhinal Cortex and Hippocampal Dentate/CA3 Underlies Age-Related Object Pattern Separation Deficits. Neuron, 97(5), 1187-1198.e4.

46. https://doi.org/10.1016/j.neuron.2018.01.039

47. Robitsek, J., Ratner, M. H., Stewart, T., Eichenbaum, H., \& Farb, D. H. (2015). Combined administration of levetiracetam and valproic acid attenuates age-related hyperactivity of CA3 place cells, reduces place field area, and increases spatial information content in aged rat hippocampus. Hippocampus, 25(12), 1541-1555. https://doi.org/10.1002/hipo.22474

48. Rolls, E. T., \& Treves, A. (1998). Neural Networks and Brain Function. Oxford University Press. https://www.oxcns.org/b3_text.html

49. Spiegel, A. M., Koh, M. T., Vogt, N. M., Rapp, P. R., \& Gallagher, M. (2013). Hilar interneuron vulnerability distinguishes aged rats with memory impairment. The Journal of Comparative Neurology, 521(15), 3508-3523. https://doi.org/10.1002/cne.23367

50. Stranahan, A. M., Haberman, R. P., \& Gallagher, M. (2011). Cognitive decline is associated with reduced reelin expression in the entorhinal cortex of aged rats. Cerebral Cortex (New York, N.Y.: 1991), 21(2), 392-400. https://doi.org/10.1093/cercor/bhq106

51. Stranahan, A. M., Salas-Vega, S., Jiam, N. T., \& Gallagher, M. (2011). Interference with reelin signaling in the lateral entorhinal cortex impairs spatial memory. Neurobiology of Learning and Memory, 96(2), 150-155. https://doi.org/10.1016/j.nlm.2011.03.009

52. Sun, Q., Sotayo, A., Cazzulino, A. S., Snyder, A. M., Denny, C. A., \& Siegelbaum, S. A. (2017). Proximodistal Heterogeneity of Hippocampal CA3 Pyramidal Neuron Intrinsic Properties, Connectivity, and Reactivation during Memory Recall. Neuron, 95(3), 656672.e3. https://doi.org/10.1016/j.neuron.2017.07.012

53. Thomé, A., Gray, D. T., Erickson, C. A., Lipa, P., \& Barnes, C. A. (2016). Memory impairment in aged primates is associated with region-specific network dysfunction. Molecular Psychiatry, 21(9), 1257-1262. https://doi.org/10.1038/mp.2015.160 
54. Tran, T., Bridi, M., Koh, M. T., Gallagher, M., \& Kirkwood, A. (2019). Reduced cognitive performance in aged rats correlates with increased excitation/inhibition ratio in the dentate gyrus in response to lateral entorhinal input. Neurobiology of Aging, 82, 120-127. https://doi.org/10.1016/j.neurobiolaging.2019.07.010

55. Tran, T., Gallagher, M., \& Kirkwood, A. (2018). Enhanced postsynaptic inhibitory strength in hippocampal principal cells in high-performing aged rats. Neurobiology of Aging, 70, 92101. https://doi.org/10.1016/j.neurobiolaging.2018.06.008

56. Treves, A., \& Rolls, E. T. (1992). Computational constraints suggest the need for two distinct input systems to the hippocampal CA3 network. Hippocampus, 2(2), 189-199. https://doi.org/10.1002/hipo.450020209

57. Tsao, A., Sugar, J., Lu, L., Wang, C., Knierim, J. J., Moser, M.-B., \& Moser, E. I. (2018). Integrating time from experience in the lateral entorhinal cortex. Nature, 561(7721), 57-62. https://doi.org/10.1038/s41586-018-0459-6

58. Wilson, I. A., Gallagher, M., Eichenbaum, H., \& Tanila, H. (2006). Neurocognitive aging: Prior memories hinder new hippocampal encoding. Trends in Neurosciences, 29(12), 662670. https://doi.org/10.1016/j.tins.2006.10.002

59. Wilson, I. A., Ikonen, S., Gallagher, M., Eichenbaum, H., \& Tanila, H. (2005). Ageassociated alterations of hippocampal place cells are subregion specific. The Journal of Neuroscience: The Official Journal of the Society for Neuroscience, 25(29), 6877-6886. https://doi.org/10.1523/JNEUROSCI.1744-05.2005

60. Witter, M. P. (2007). Intrinsic and extrinsic wiring of CA3: Indications for connectional heterogeneity. Learning \& Memory (Cold Spring Harbor, N.Y.), 14(11), 705-713. https://doi.org/10.1101//m.725207

61. Wood, E. R., Dudchenko, P. A., Robitsek, R. J., \& Eichenbaum, H. (2000). Hippocampal neurons encode information about different types of memory episodes occurring in the same location. Neuron, 27(3), 623-633. https://doi.org/10.1016/s0896-6273(00)00071-4

62. Yassa, M. A., Mattfeld, A. T., Stark, S. M., \& Stark, C. E. L. (2011). Age-related memory deficits linked to circuit-specific disruptions in the hippocampus. Proceedings of the National Academy of Sciences of the United States of America, 108(21), 8873-8878. https://doi.org/10.1073/pnas.1101567108

63. Yassa, M. A., Stark, S. M., Bakker, A., Albert, M. S., Gallagher, M., \& Stark, C. E. L. (2010). High-resolution structural and functional MRI of hippocampal CA3 and dentate gyrus in patients with amnestic Mild Cognitive Impairment. Neurolmage, 51(3), 1242-1252. https://doi.org/10.1016/j.neuroimage.2010.03.040 

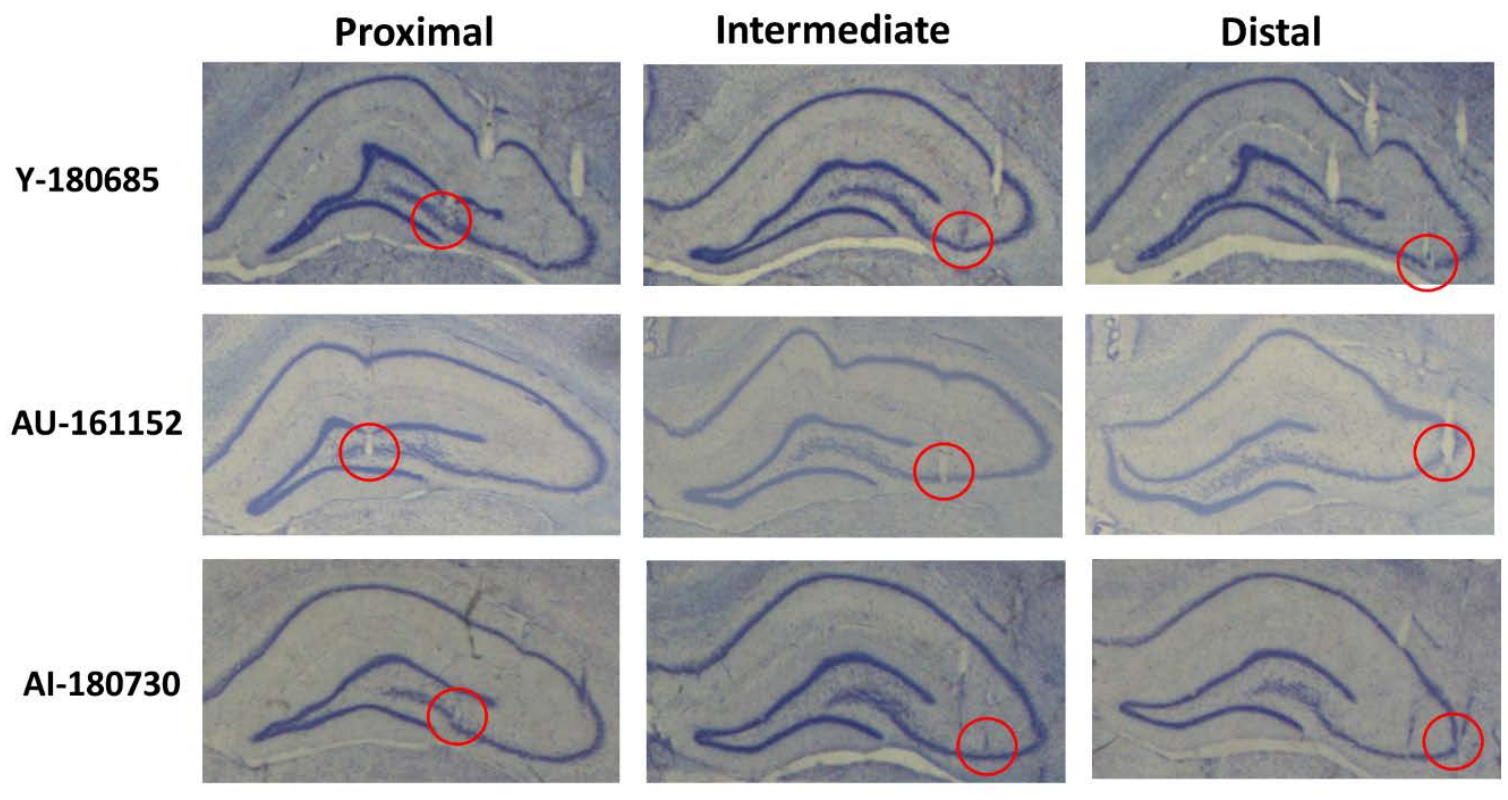

Supp. S1. Examples of histological verification of tetrode tracks in CA3. Example rat from $Y$, AU, Al groups with tetrodes in all CA3 subregions. Tetrode tracks were verified on Nissl-stained coronal sections. Tetrode tips are indicated by red circles. 
A)

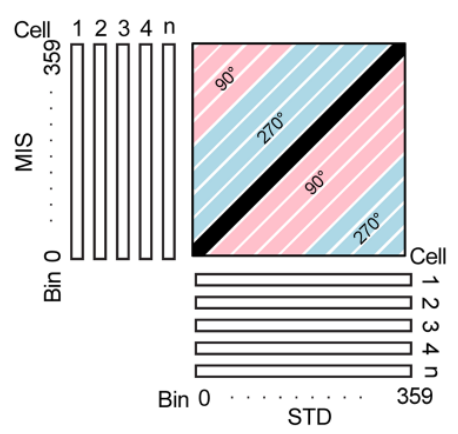

Norm sum corr diff $=\frac{\sum \text { STDv }-\sum \text { MISv }}{\sum \text { STDv }+\sum \text { MISv }}$

STDv = correlation values along the main diagonal in the STD1 X STD2 matrix MISv = correlation values along the main diagonal in the STD1 X MIS matrix

B)
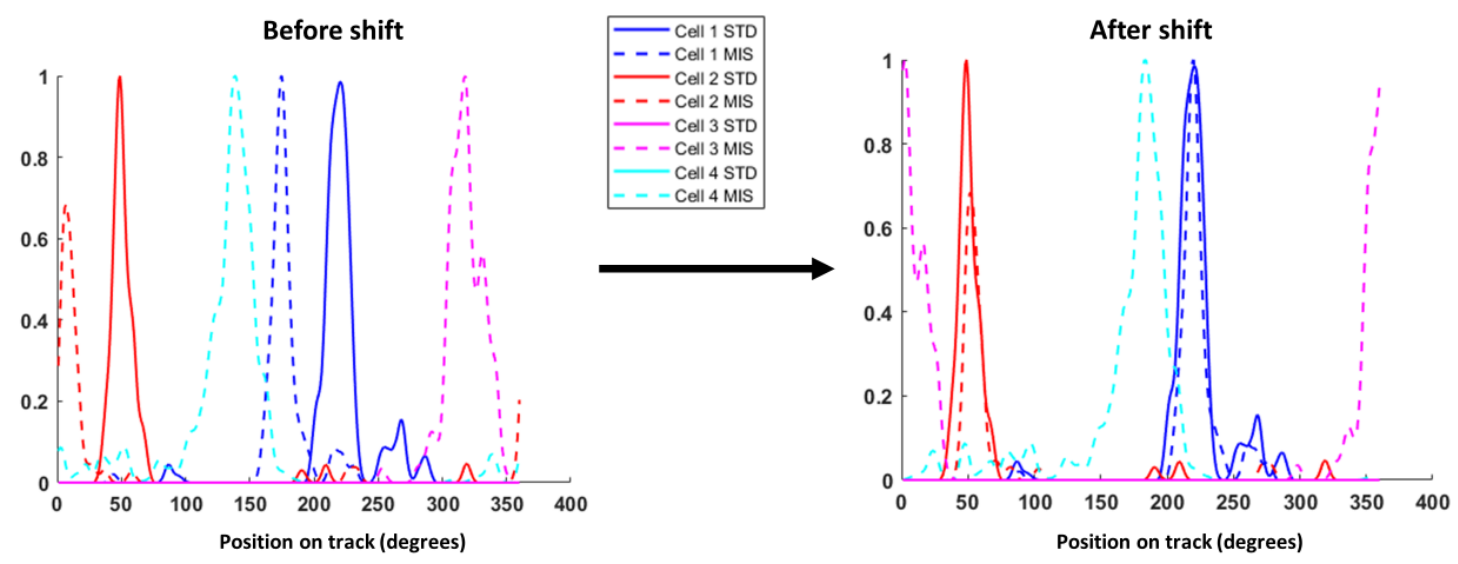

Supp. S2. (A) Illustration showing the population correlation analysis for the STD vs. MIS sessions. To create these matrices, the firing rate of each cell was calculated for each $1^{\circ}$ bin on the track and normalized to its peak rate. The firing rate maps of all $n$ cells in the sample were stacked to create a $360 \times n$ matrix, in which each column of the matrix represents the population firing rate vector for each angle of the track. The firing rate vectors at each angle of a STD session (STD1) were correlated with the firing rate vectors at each angle of the next STD session (STD2), to create STD1 x STD2 correlation matrices, or with the next MIS session, to create STD1 $x$ MIS correlation matrices. The normalized correlation difference was calculated as the difference between the sum of the correlation values along the main diagonal in the STD1 $x$ STD2 matrix (STDV) and the sum of the correlation values along the main diagonal in the STD1 x MIS matrix (MISv), divided by the sum of these two quantities. (B) To align the place fields in each MIS session to the place fields in the preceding STD session, each cell's firing rate map was rotated by the mean rotation amount of each MIS session. In this example, Cell 1 (blue) and cell 2 (red) are Rotate cells (i.e., they have place fields in both STD and MIS sessions) and cell 3 (magenta) and cell 4 (cyan) are Remap cells (i.e., place fields appeared in MIS session but are silent in STD session). Before the shift (left), cell 1 fired at the $210^{\circ}$ location on the track in the STD session (blue, solid line) but fired at the $170^{\circ}$ location in the MIS session (blue, dotted line). Similarly, cell 2 fired at the $50^{\circ}$ location in the STD session (red, solid line) but fired at the $10^{\circ}$ location in the MIS session (red, dotted line). Cell 3 (magenta, 
dotted line) and cell 4 (cyan, dotted line) fired in the MIS session but they did not fire in the STD sessions. For this example, the mean rotation amount was $40^{\circ}$ in the $\mathrm{CCW}$ direction. After shifting the place fields in the MIS session by the mean rotation amount (right), the place fields of cell 1 and cell 2 in the MIS session fired at the same location as the STD session. The place fields of cell 3 and cell 4 were also rotated by the mean rotation amount. Thus, shifting aligned the place fields of Rotate cells in the STD and MIS sessions, which allowed different experimental mismatch angles to be collapsed into one MIS session. 

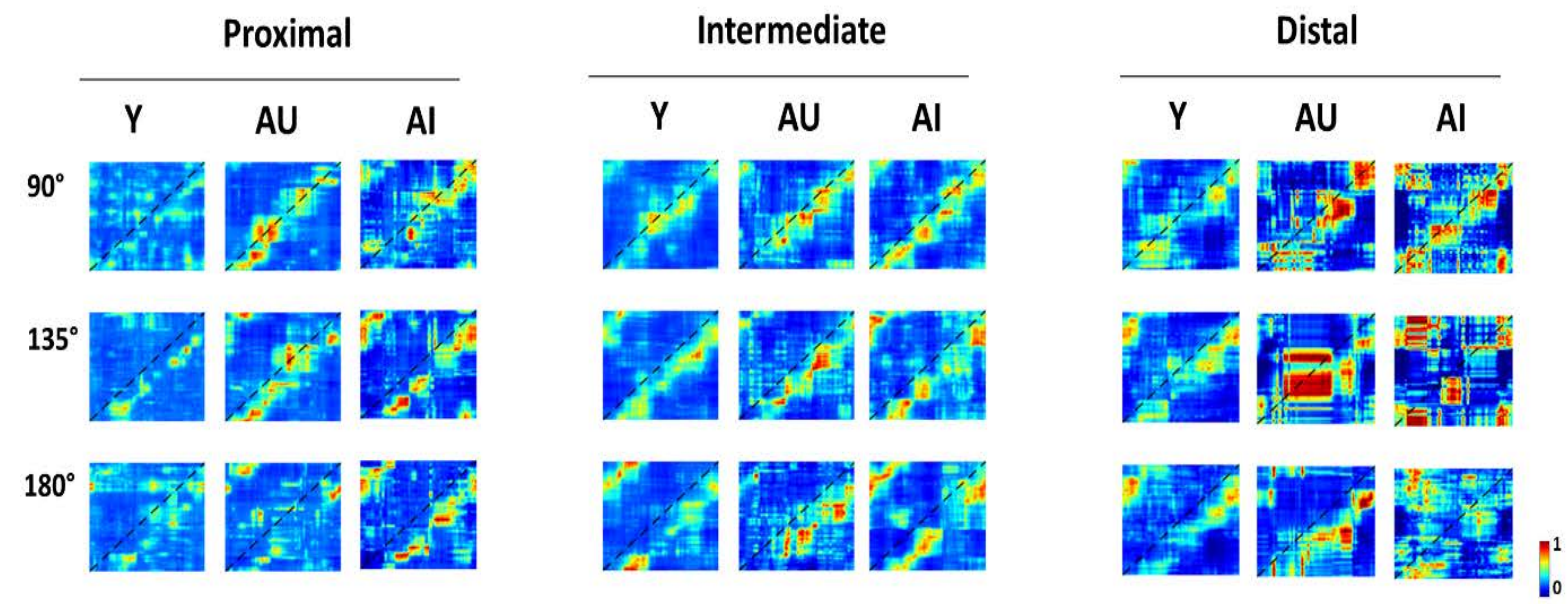

Supp. S3. STD1 x MIS correlation matrices for each mismatch angle. In agreement with prior studies (H. Lee et al., 2015; I. Lee et al., 2004; Neunuebel \& Knierim, 2014), the place fields tended to rotate CCW by the amount of the local-cue rotation, causing bands of higher correlation below the main diagonal (dashed line) in the STD1 x MIS correlation matrices for each mismatch amount. As the mismatch angle increased, the correlation band moved further away from the main diagonal. Note that this effect is not clearly evident in all matrices, partially due to small sample sizes from distal CA3. Because of the variability, the data from all 3 mismatch angles were collapsed for the analyses in the main text. 
A)
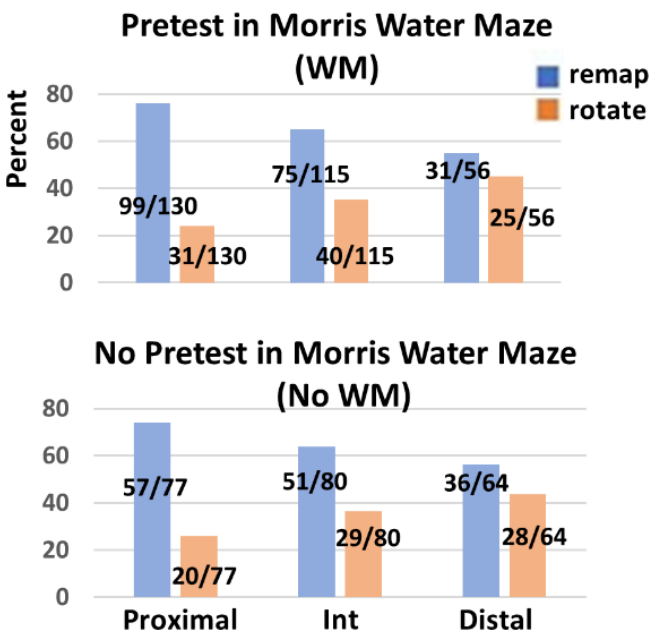

B)

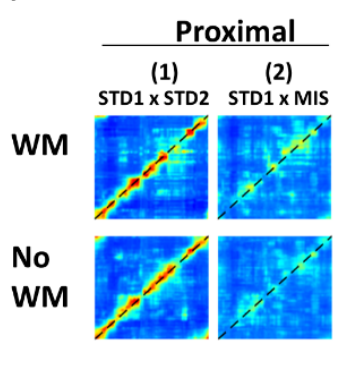

Intermediate

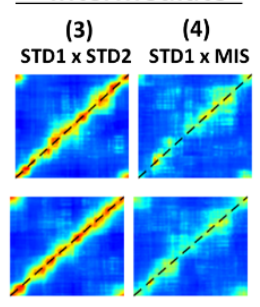

Distal

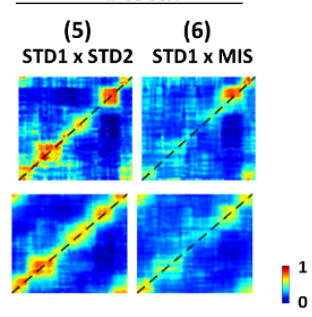

C)

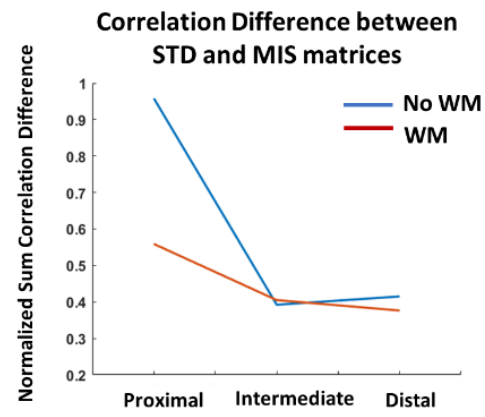

Supp. S4. Because 4 out of $8 Y$ rats in the cue-mismatch experiment were from a previous study (H. Lee et al., 2015) that were not pretested in the Morris water maze (see Methods), data from two $Y$ groups were compared. (A) Classification of responses in CA3 subregions were not different between the $4 \mathrm{Y}$ rats pretested in the Morris water maze (WM) and the $4 \mathrm{Y}$ rats not pretested in the Morris Water Maze (No WM). There was a similar trend of a decreasing proportion of Remap cells from proximal to distal CA3, with an increasing proportion of Rotate cells from proximal to distal CA3. (B) STD1 x STD2 matrices (columns 1, 3, 5) and STD1 x MIS matrices (columns 2, 4, 6) in each CA3 subregions. (C) The correlation differences between STD and MIS matrices showed a decreasing trend from proximal to distal CA3 in both WM and No WM groups. The strengths of the correlation differences in the intermediate and distal regions were not different between the WM and No WM groups, but the strength of the correlation difference was higher in the No WM than the WM group in the proximal region. Despite the numerically greater correlation difference in proximal CA3, inclusion of the No WM group did not alter the conclusions of the study (Supp Fig S5). 
A)

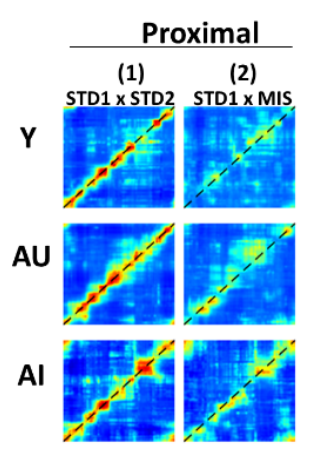

Intermediate

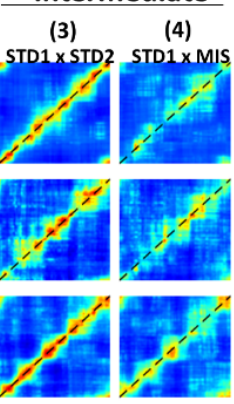

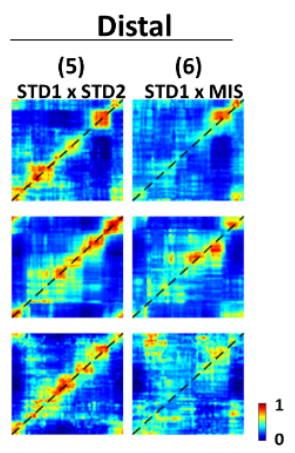

B)

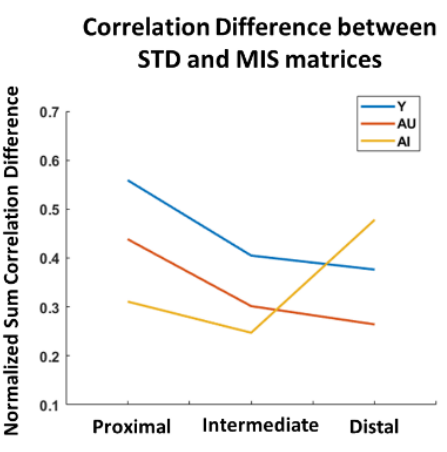

C)

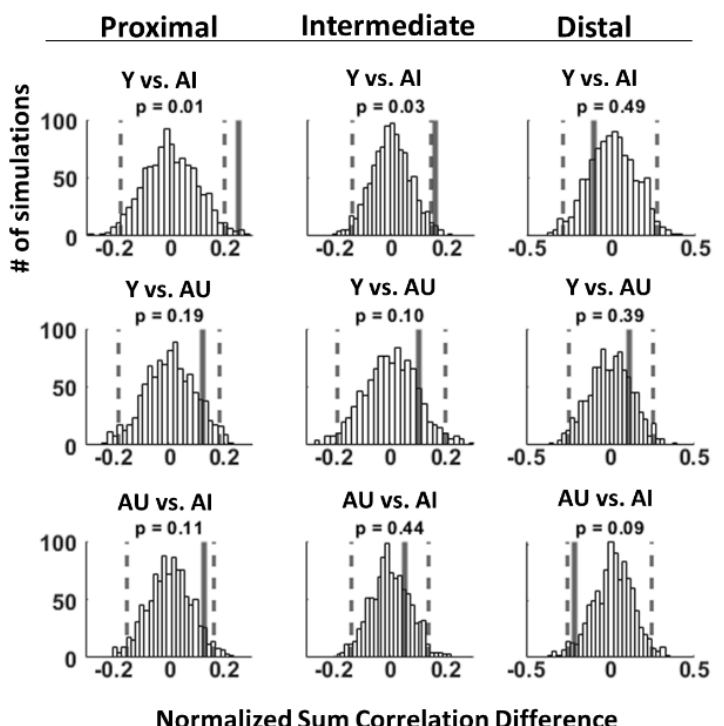

D)

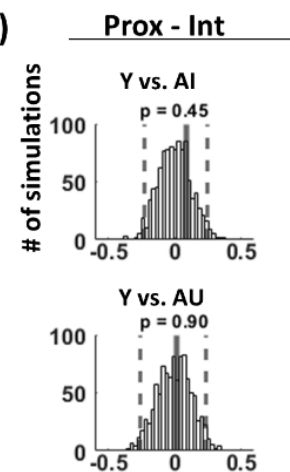

Int - Dist Prox - Dist
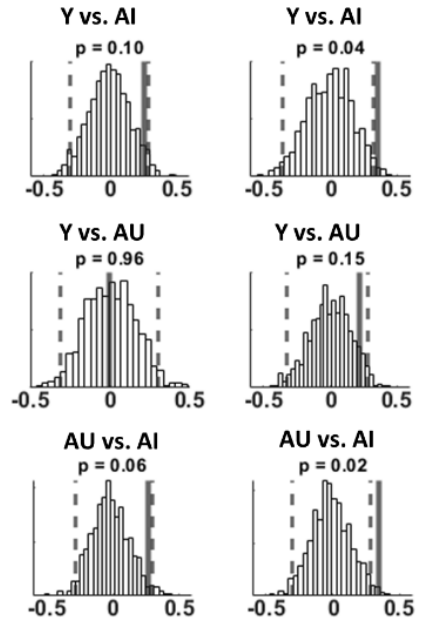

Y vs. AU

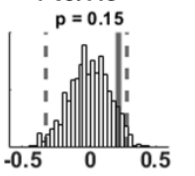

AU vs. Al

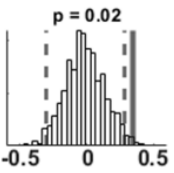

Normalized Sum Correlation Difference

Supp. S5. Because 4 out of $8 Y$ rats in the cue-mismatch experiment were from a previous study $(\mathrm{H}$. Lee et al., 2015) and (see Supp. S4), data from only the $4 \mathrm{Y}$ rats recorded for the current study were compared with the aged rats to test whether the overall results changed. (A) STD1 x STD2 matrices (columns 1, 3, 5) and STD1 x MIS matrices (columns 2, 4, 6) in each CA3 subregions for all age groups. (B) The correlation difference between STD and MIS matrices showed a decreasing trend in $Y$ and $A U$ rats from proximal to distal $C A 3$ but an increasing trend in Al rats. (C) Comparisons between the age groups were calculated for each subregion (top row: $\mathrm{Y}-\mathrm{Al}$; middle: $\mathrm{Y}-\mathrm{AU}$; bottom: $\mathrm{AU}-\mathrm{Al}$ ). The observed value (thick, black line) was compared to the distribution produced by 1,000 random shuffles of the data. (D) To test for interactions between age and subregions, comparisons between the subregions were calculated for each between-age group comparisons. The correlation differences between $\mathrm{Y}$ and aged rats were not significant at the significance level $\alpha=0.005$ with Bonferroni correction for 9 comparisons. However, the overall patterns of data (the comparisons that show low $p<$ $0.05)$ are consistent with the results in Fig. 3, in that $Y$ rats show differences from Al rats in proximal and intermediate regions (C) and interactions between proximal and distal regions (D). Thus, although the statistical evidence is somewhat weaker with the exclusion of the 4 rats from the previous study, the pattern of results is not altered (compared with Fig. 3). 
A)

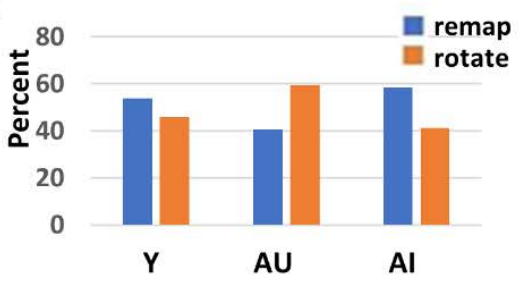

B)

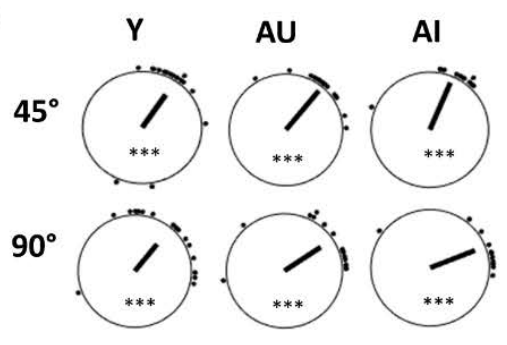

C)
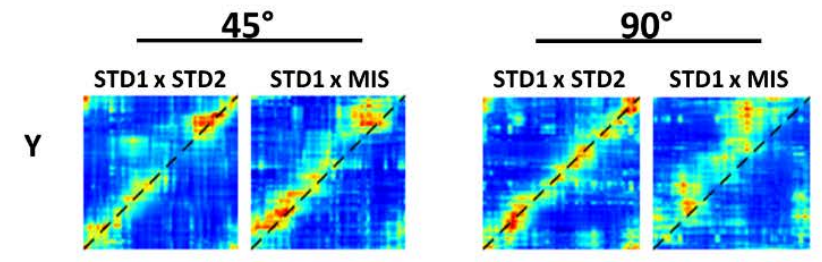

AU
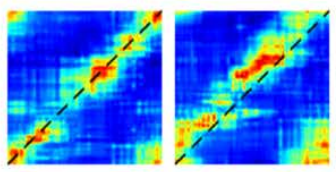

Al

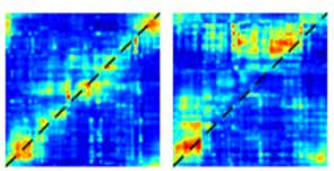

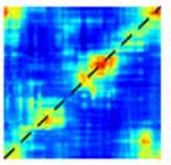
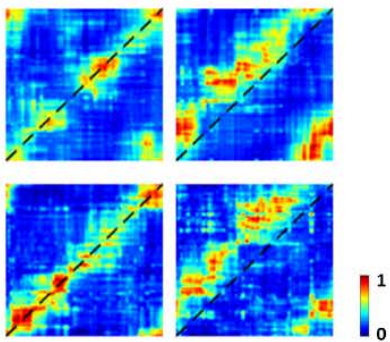

Supp. S6. Population coherence demonstrates control by the global cues in all age groups when local cues are removed. (A) When local texture cues were removed and only global cues were rotated by $45^{\circ}$ or $90^{\circ}$, the proportion of cells that Rotate or Remap did not differ significantly in all age groups $\left(X^{2}=4.13, p=0.13\right)$. Cells from all CA3 subregions were pooled together. (B) Individual rotation amounts show significant clustering, although the rotation of the place fields was less than that of the cues, demonstrating that there were uncontrolled cues or influences of path integration that conflicted with the controlled cue set. [Rayleigh test, for $45^{\circ}$ : $\mathrm{Y}: \mathrm{n}=16, \mathrm{z}=8.05, \mathrm{p}<0.0001 ; \mathrm{AU}: \mathrm{n}=19, \mathrm{z}=15.76, \mathrm{p}<0.0001 ; \mathrm{Al}: \mathrm{n}=12, \mathrm{z}=9.49, \mathrm{p}<$ 0.0001; for 90: Y: $n=19, z=7.33, p<0.0001 ; A U: n=18, z=10.14, p<0.0001 ; A l: n=12, z$ $=7.9, p<0.0001]^{* \star *}$ denotes $p<0.0001$. (C) Spatial correlation matrices show that the population maintained coherence in all age groups, as indicated by the bands of high correlation. The bands of correlation in the STD1 x MIS matrices shifted upward (above the dotted diagonal line), indicating control by the global cues in all age groups. 


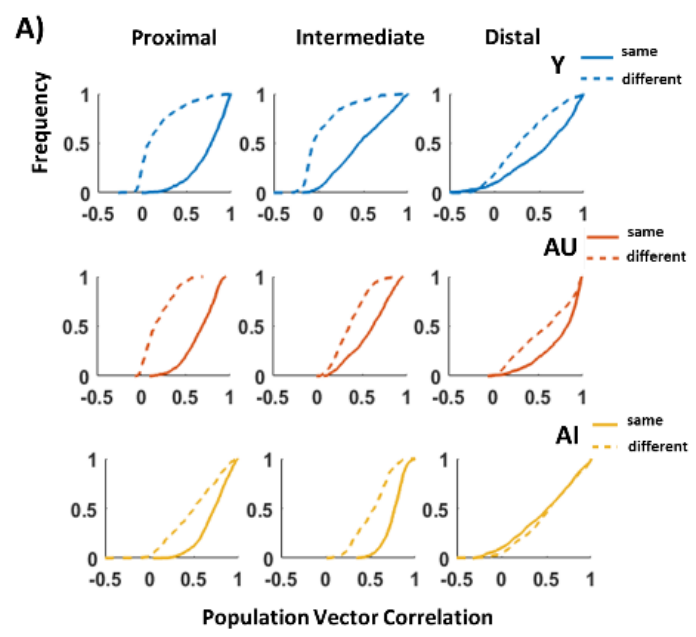

B)

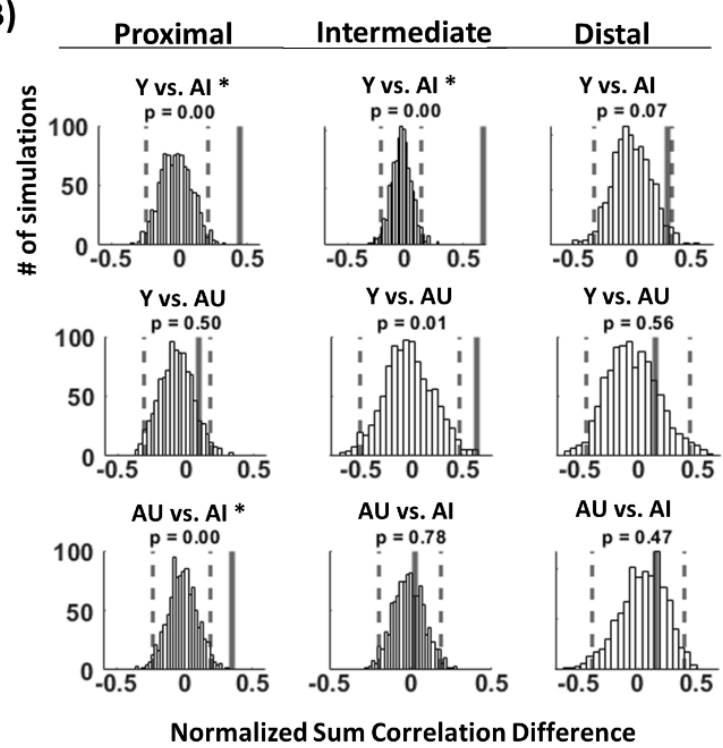

C)

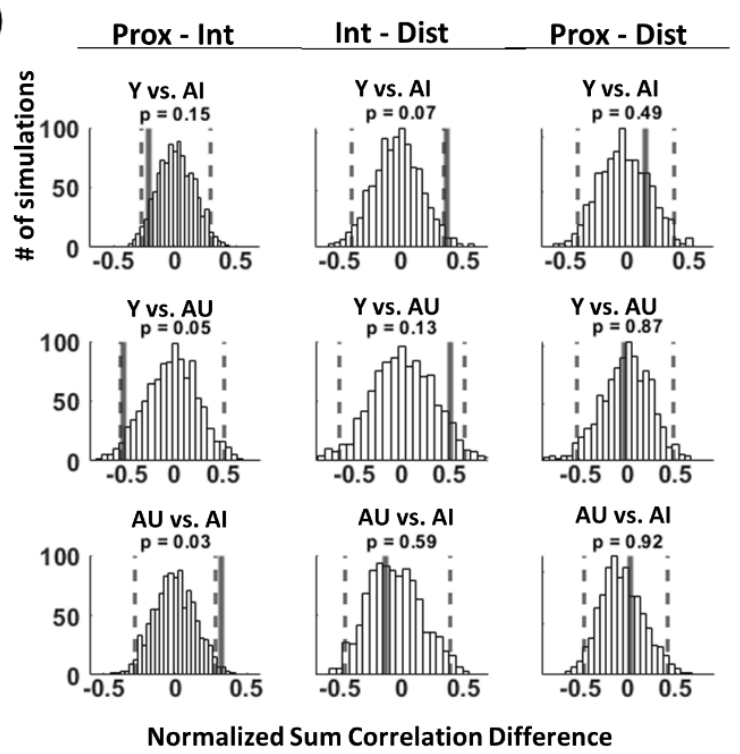

Supp. S7. For $Y$ rats, the PV correlation for the same shapes, particularly in proximal and intermediate CA3, showed less correlation than both AU and AI rats (Fig. 5B). Of the $3 \mathrm{Y}$ rats recorded in the two-shape experiment, $1 \mathrm{Y}$ rat showed an anomalous remapping between the same shapes (i.e., the place field locations changed between visits to the same shapes). Although the anomalous rat was included in the main analysis, approximately $47 \%$ (53/112) of the total cell sample in $Y$ rats was from that rat. To determine whether removing the outlier rat changes the remapping differences observed between $Y$ rats and aged rats, the anomalous rat was excluded from the $\mathrm{Y}$ data set. Removing the outlier rat increased the PV correlations for the same shape and altered the statistical outcomes of the interactions, but it did not change the overall pattern of remapping differences observed between $Y$ rats and aged rats (compare with Fig. 5). (A) Cumulative distribution plots for the PV correlations between the same shape (solid lines) and the different shape (dotted lines). Removing the outlier $Y$ rat increased the PV correlations between the same shape. (B) Shuffling analysis comparing the PV correlation differences between the animal groups in each CA3 subregion showed that similar to Fig. 5, Y and $\mathrm{AU}$ rats showed more remapping in proximal but similar degree of remapping in distal CA3 
bioRxiv preprint doi: https://doi.org/10.1101/2021.09.14.460329; this version posted September 16, 2021. The copyright holder for this preprint (which was not certified by peer review) is the author/funder. All rights reserved. No reuse allowed without permission.

compared to Al rats. $\mathrm{Y}$ rats also showed more remapping in intermediate CA3 compared to Al and $A U$ rats. (C) There were no significant age group and subregion interactions, although trends towards significance between $Y$ and aged rats in the subregions were similar to Fig 5 . ( ${ }^{*}$ denotes significance at $p=0.005$, Bonferroni corrected for 9 comparisons) 


\begin{tabular}{|l|c|c|c|c|c|c|}
\hline & \multicolumn{2}{|c|}{ Proximal } & \multicolumn{2}{c|}{ Intermediate } & \multicolumn{2}{c|}{ Distal } \\
\hline Rat & Remap & Rotate & Remap & Rotate & Remap & Rotate \\
\hline Y-409 & 7 & 5 & 4 & 5 & 13 & 14 \\
\hline Y-434 & 3 & 0 & 24 & 12 & 1 & 3 \\
\hline Y-459 & 17 & 1 & 17 & 8 & 8 & 5 \\
\hline Y-460 & 30 & 14 & 6 & 4 & 14 & 6 \\
\hline Y-160281 & 6 & 3 & 3 & 3 & 6 & 14 \\
\hline Y-160517 & 7 & 9 & 8 & 7 & & \\
\hline Y-180576 & 52 & 7 & 45 & 19 & & \\
\hline Y-180685 & 34 & 12 & 19 & 11 & 25 & 11 \\
\hline AU-151055 & 9 & 13 & 9 & 16 & & \\
\hline AU-160782 & 2 & 1 & 4 & 3 & & \\
\hline AU-161152 & 19 & 1 & 18 & 17 & 0 & 2 \\
\hline AU-170654 & 10 & 7 & 6 & 8 & 12 & 18 \\
\hline AU-170711 & 14 & 11 & 3 & 2 & 1 & 5 \\
\hline AU-190230 & 10 & 14 & 11 & 10 & 5 & 3 \\
\hline AU-190268 & 17 & 14 & 1 & 1 & & \\
\hline Al-151170 & 8 & 21 & & & & \\
\hline Al-161201 & 2 & 5 & 5 & 6 & & \\
\hline Al-170869 & & & 2 & 10 & 3 & 4 \\
\hline Al-180730 & 2 & 16 & 4 & 8 & 0 & 1 \\
\hline Al-180821 & & & 0 & 1 & 6 & 10 \\
\hline Al-190127 & 12 & 19 & 23 & 42 & 1 & 1 \\
\hline Al-190196 & & & 24 & 40 & 10 & 11 \\
\hline
\end{tabular}

Table S1. Individual rat responses to the cue-mismatch manipulations. The CA3 analyses in the main text combined data across multiple sessions from $8 \mathrm{Y}, 7 \mathrm{AU}$, and $7 \mathrm{Al}$ rats. The total number of cell responses to the cue-mismatch manipulations observed from tetrodes in proximal, intermediate, and distal subregions for individual rats are listed. 


\begin{tabular}{|l|c|c|c|c|c|c|}
\hline & \multicolumn{2}{|c|}{ Proximal } & \multicolumn{2}{c|}{ Intermediate } & \multicolumn{2}{c|}{ Distal } \\
\hline Rat & Shape & Room & Shape & Room & Shape & Room \\
\hline Y-160517 & 10 & 12 & 3 & 6 & & \\
\hline Y-180576 & 27 & 15 & 26 & 24 & & \\
\hline Y-180685 & 23 & 29 & 14 & 13 & 9 & 12 \\
\hline AU-151055 & 17 & 16 & 15 & 19 & 2 & 2 \\
\hline AU-161152 & 14 & 11 & 6 & 9 & & \\
\hline AU-170654 & 7 & 6 & 5 & 7 & 17 & 18 \\
\hline AU-170711 & 6 & 5 & 1 & 1 & 2 & 3 \\
\hline AU-190230 & 6 & 9 & 15 & 16 & 2 & 4 \\
\hline AU-190268 & 20 & 13 & 7 & 7 & & \\
\hline Al-151170 & 12 & 15 & & & & \\
\hline Al-161201 & 4 & 6 & 5 & 5 & & \\
\hline Al-170869 & & & 9 & 4 & 1 & \\
\hline Al-180730 & 10 & 7 & 2 & 2 & & \\
\hline Al- 190127 & 13 & 14 & 52 & 53 & 2 & 2 \\
\hline Al-190196 & & & 16 & 16 & 5 & 3 \\
\hline
\end{tabular}

Table S2. Total number of cells from individual rats during the two-shape experiment (Shape) and the two-room experiment (Room). The CA3 analyses in the main text combined data across multiple sessions from $3 \mathrm{Y}, 6 \mathrm{AU}$, and $6 \mathrm{Al}$ rats. The total number of cells recorded from tetrodes in proximal, intermediate, and distal subregions for individual rats are listed 